\title{
DIFFUSIVE TRANSPORT OF PARTIALLY QUANTIZED PARTICLES: EXISTENCE, UNIQUENESS AND LONG-TIME BEHAVIOUR
}

\author{
N. BEN ABDALLAH, F. MÉHATS AND N. VAUCHELET \\ Mathématiques pour l'Industrie et la Physique (UMR 5640), Université Paul Sabatier, \\ 118 route de Narbonne, 31062 Toulouse cedex 4, France \\ (naoufel@mip.ups-tlse.fr; mehats@mip.ups-tlse.fr; vauchel@mip.ups-tlse.fr)
}

(Received 20 September 2004)

\begin{abstract}
A self-consistent model for charged particles, accounting for quantum confinement, diffusive transport and electrostatic interaction is considered. The electrostatic potential is a solution of a threedimensional Poisson equation with the particle density as the source term. This density is the product of a two-dimensional surface density and that of a one-dimensional mixed quantum state. The surface density is the solution of a drift-diffusion equation with an effective surface potential deduced from the fully three-dimensional one and which involves the diagonalization of a one-dimensional Schrödinger operator. The overall problem is viewed as a two-dimensional drift-diffusion equation coupled to a SchrödingerPoisson system. The latter is proven to be well posed by a convex minimization technique. A relative entropy and an a priori $L^{2}$ estimate provide sufficient bounds to prove existence and uniqueness of a global-in-time solution. In the case of thermodynamic equilibrium boundary data, a unique stationary solution is proven to exist. The relative entropy allows us to prove the convergence of the transient solution towards it as time grows to infinity. Finally, the low-order approximation of the relative entropy is used to prove that this convergence is exponential in time.
\end{abstract}

Keywords: Schrödinger equation; drift-diffusion system; relative entropy; long-time behaviour; subband method; convex minimization

2000 Mathematics subject classification: Primary 35Q40; 76R99; 74H40; 49S05; 49K20

\section{Introduction and main result}

The drift-diffusion equation is one of the most used models for charged particle transport in various areas such as gas discharges, plasmas or semiconductors. It consists in a conservation equation for the particle density, in which the current density is the sum of two terms. One is proportional to the particle density and to the electrostatic forces. This term is referred to as the drift current. The second term is the diffusion current and is proportional to the gradient of the particle density $[\mathbf{9}, \mathbf{1 0}, \mathbf{1 8}, \mathbf{1 9}, \mathbf{2 5}, \mathbf{2 6}]$.

The drift-diffusion model can be derived from kinetic theory when the mean-free path related to particle interactions with a thermal bath is small compared with the system length scale. In semiconductors, one of the most important mechanisms driving the electrons towards a diffusive regime is collisions with phonons (vibrations of the semiconductor crystal lattice) which drive the electrons towards a local equilibrium at the lattice 
temperature $[\mathbf{2 5}, \mathbf{3 2}]$. We refer the reader to $[\mathbf{2 1}, \mathbf{3 1}]$ for a rigorous derivation from the Boltzmann equation and to $[\mathbf{1 8}, \mathbf{1 9}, \mathbf{2 5}, \mathbf{2 6}]$ and references therein for the analysis of this system when coupled to the Poisson equation for the electrostatic potential.

Quantum systems at global thermodynamic equilibrium can be described as a statistical mixture of eigenstates of the Schrödinger operator. The occupation number of each state is given by a thermodynamic equilibrium statistic function. Typically, it is given by

$$
\exp \left(\frac{E_{\mathrm{F}}-E}{k_{\mathrm{B}} T}\right)
$$

for Boltzmann statistics or

$$
\left(1+\exp \left(\frac{E-E_{\mathrm{F}}}{k_{\mathrm{B}} T}\right)\right)^{-1}
$$

for Fermi-Dirac statistics, where $E$ is the energy of the considered state, $k_{\mathrm{B}}$ is the Boltzmann constant, $T$ is the temperature and $E_{\mathrm{F}}$ is the so-called Fermi energy which, at zero temperature, represents the threshold between occupied and unoccupied states $[27-29,35]$.

In nanoscale semiconductor devices like ultrashort channel double gate MOSFETs (DGMOSs), electrons might be extremely confined in one of several directions that we shall refer to as the confining directions. This leads to a partial quantization of the energy. In the non-confined direction(s), which we shall also refer to as the transport direction(s), following the length and energy scales, transport might have a quantum nature or be purely classical in the kinetic or diffusive regimes. In the present work, we are interested in the last regime. Namely, we consider a particle system which is partially quantized in one direction (denoted by $z$ ) and which, in the transport direction denoted by $x$, has a diffusive motion. The system is at equilibrium in the confined direction with a local Fermi level $\epsilon_{F}$ which depends on the transport variable $x$. The variable $x$ is assumed to lie in a bounded regular domain $\omega \in \mathbb{R}^{2}$ while $z$ belongs to the interval $(0,1)$. The spatial domain is then $\Omega=\omega \times(0,1)$. At a time $t$ and a position $(x, z)$, the particle density $N(t, x, z)$ is given by

$$
N(t, x, z)=\sum_{k=1}^{+\infty} \mathrm{e}^{\epsilon_{F}(t, x)-\epsilon_{k}(t, x)}\left|\chi_{k}(t, x, z)\right|^{2},
$$

where $\epsilon_{F}$ is the Fermi level and $\left(\chi_{k}, \epsilon_{k}\right)$ is the complete set of eigenfunctions and eigenvalues of the Schrödinger operator in the $z$-variable:

$$
\left.\begin{array}{c}
-\frac{1}{2} \partial_{z}^{2} \chi_{k}+V \chi_{k}=\epsilon_{k} \chi_{k}, \quad k \geqslant 1, \\
, x, \cdot) \in H_{0}^{1}(0,1), \quad \int_{0}^{1} \chi_{k} \chi_{\ell} \mathrm{d} z=\delta_{k \ell} .
\end{array}\right\}
$$

The electrostatic potential $V$ is a solution of the Poisson equation

$$
-\Delta_{x, z} V=N
$$


The surface density

$$
N_{\mathrm{S}}(t, x)=\int_{0}^{1} N(t, x, z) \mathrm{d} z=\mathrm{e}^{\epsilon_{F}} \sum_{k=1}^{+\infty} \mathrm{e}^{-\epsilon_{k}(t, x)}
$$

satisfies the drift-diffusion equation

$$
\partial_{t} N_{\mathrm{s}}-\operatorname{div}_{x}\left(\nabla_{x} N_{\mathrm{s}}+N_{\mathrm{s}} \nabla_{x} V_{\mathrm{s}}\right)=0,
$$

where the effective potential $V_{\mathrm{s}}$ is given by

$$
V_{\mathrm{s}}=-\log \sum_{k} \mathrm{e}^{-\epsilon_{k}} .
$$

Note also that $N$ can be rewritten as

$$
N(t, x, z)=\frac{N_{\mathrm{s}}(t, x)}{\mathcal{Z}(t, x)} \sum_{k=1}^{+\infty} \mathrm{e}^{-\epsilon_{k}(t, x)}\left|\chi_{k}(t, x, z)\right|^{2},
$$

where the repartition function $\mathcal{Z}$ is given by

$$
\mathcal{Z}(t, x)=\sum_{k=1}^{+\infty} \mathrm{e}^{-\epsilon_{k}(t, x)} .
$$

The unknowns of the overall system are the surface density $N_{\mathrm{s}}(t, x)$, the eigenenergies $\epsilon_{k}(t, x)$, the eigenfunctions $\chi_{k}(t, x, z)$ and the electrostatic potential $V(t, x, z)$. The Fermi level $\epsilon_{F}$ is determined by

$$
\epsilon_{F}(t, x)=\log \frac{N_{\mathrm{s}}(t, x)}{\mathcal{Z}(t, x)} .
$$

This will be useful for the study of global equilibria. The system (1.2)-(1.8) is completed with the initial condition

$$
N_{\mathrm{s}}(0, x)=N_{\mathrm{s}}^{0}(x)
$$

and with the following boundary conditions:

$$
\left.\begin{array}{cl}
N_{\mathrm{s}}(t, x)=N_{b}(x), \quad V(t, x, z)=V_{b}(x, z), & \text { for } x \in \partial \omega, z \in(0,1), \\
\partial_{z} V(t, x, 0)=\partial_{z} V(t, x, 1)=0, & \text { for } x \in \omega
\end{array}\right\}
$$

In applications like the DGMOS [4], the frontier $\partial \omega \times[0,1]$ includes the source and the drain contacts as well as an insulating or artificial boundary. On the other hand, $\omega \times\{0\}$ and $\omega \times\{1\}$ represent the gate contacts (in addition to possible insulating boundaries). Mixed-type boundary conditions are then to be prescribed. The boundary conditions (1.11) do not take into account this complexity and are chosen for mathematical convenience: elliptic regularity properties of the Poisson equation (1.3) are needed in our proofs. 


\subsection{Main results}

\section{Assumption 1.1.}

(i) The initial condition satisfies $N_{\mathrm{s}}^{0} \in L^{2}(\omega)$ and $N_{\mathrm{s}}^{0} \geqslant 0$, a.e.

(ii) The boundary data for the surface density satisfy $0<N_{1} \leqslant N_{b} \leqslant N_{2}$ a.e., where $N_{1}$ and $N_{2}$ are positive constants and $N_{b} \in C^{2}(\partial \omega)$.

(iii) The Dirichlet datum for the potential satisfies $V_{b} \in C^{2}(\partial \omega \times[0,1])$ and the compatibility condition

$$
\frac{\partial V_{b}}{\partial z}(x, 0)=\frac{\partial V_{b}}{\partial z}(x, 1)=0, \quad \forall x \in \partial \omega
$$

The first result of this paper is the following existence and uniqueness theorem.

Theorem 1.2. Let $T>0$ be fixed. Under Assumption 1.1, the system (1.2)-(1.11) admits a unique weak solution such that

$$
N_{\mathrm{s}} \in C\left([0, T], L^{2}(\omega)\right) \cap L^{2}\left((0, T), H^{1}(\omega)\right), \quad V \in C\left([0, T], H^{2}(\Omega)\right) .
$$

The second result concerns the asymptotic behaviour of the solution as $t \rightarrow \infty$. To this aim, we shall first define the notion of global equilibrium for boundary data, under which we show that there exists a unique stationary solution, and finally prove that the time-dependent solution converges exponentially fast to this stationary solution.

Assumption 1.3. The boundary is said to be at global equilibrium if there exists a real number $u^{\infty}>0$ such that $\forall x \in \partial \omega, N_{b}(x)=u^{\infty} \mathrm{e}^{-V_{\mathrm{s}}^{\infty}(x)}$, where $V_{\mathrm{s}}^{\infty}$ is defined by

$$
V_{\mathrm{S}}^{\infty}(x)=-\log \left(\sum_{k} \mathrm{e}^{-\epsilon_{k}\left[V_{b}\right](x)}\right) .
$$

In view of (1.9), it means that the Fermi level at the boundary is constant.

In this assumption, as well as in the remainder of the paper, for each potential $V$, the notation $\epsilon_{k}[V]$ stands for the $k$ th eigenvalue of the Hamiltonian $-\frac{1}{2} \partial_{z}^{2}+V$ and $\chi_{k}[V]$ denotes the corresponding eigenfunction (solving (1.2)).

The stationary problem reads

$$
\left.\begin{array}{c}
-\operatorname{div}_{x}\left(\nabla_{x} N_{\mathrm{s}}^{\infty}+N_{\mathrm{s}}^{\infty} \nabla_{x} V_{\mathrm{s}}^{\infty}\right)=0, \\
-\frac{1}{2} \partial_{z}^{2} \chi_{k}^{\infty}+V^{\infty} \chi_{k}^{\infty}=\epsilon_{k}^{\infty} \chi_{k}^{\infty}, \\
-\Delta_{x, z} V^{\infty}=N^{\infty}=\frac{N_{\mathrm{s}}^{\infty}}{\mathcal{Z}^{\infty}} \sum_{k=1}^{+\infty}\left|\chi_{k}^{\infty}\right|^{2} \mathrm{e}^{-\epsilon_{k}^{\infty}}, \\
\mathcal{Z}^{\infty}=\sum_{\ell=1}^{+\infty} \mathrm{e}^{-\epsilon_{\ell}^{\infty}},
\end{array}\right\}
$$


with the boundary conditions

$$
\left.\begin{array}{rl}
N_{\mathrm{s}}^{\infty}(x)=N_{b}(x), \quad V^{\infty}(x, z)=V_{b}(x, z) & \text { for } x \in \partial \omega, z \in(0,1), \\
\partial_{z} V^{\infty}(x, 0)=\partial_{z} V^{\infty}(x, 1)=0 & \text { for } x \in \omega,
\end{array}\right\}
$$

where we have used the shorthand notation $\epsilon_{k}^{\infty}$ for $\epsilon_{k}\left[V^{\infty}\right]$ and $\chi_{k}^{\infty}$ for $\chi_{k}\left[V^{\infty}\right]$.

Proposition 1.4. Under Assumptions 1.1 and 1.3, the stationary problem (1.12), (1.13) admits a unique solution such that $N_{\mathrm{s}}^{\infty} \in C^{2}(\bar{\omega})$ and $V^{\infty} \in C^{2}(\bar{\Omega})$.

The following theorem proves the exponential convergence of the time-dependent solution towards the stationary one.

Theorem 1.5. Let Assumptions 1.1 and 1.3 hold. Let $N_{\mathrm{s}}, V$ and $N_{\mathrm{s}}^{\infty}, V^{\infty}$ respectively be the time-dependent and the stationary solutions defined respectively in Theorem 1.2 and Proposition 1.4. There exist two constants $\lambda>0$ and $C>0$ such that for all $t \geqslant 0$,

$$
\left\|N_{\mathrm{s}}-N_{\mathrm{s}}^{\infty}\right\|_{L^{2}(\omega)}(t)+\left\|V-V^{\infty}\right\|_{H^{1}(\omega)}(t) \leqslant C \mathrm{e}^{-\lambda t} .
$$

The outline of the paper is as follows. In the next subsection, we briefly explain how the drift-diffusion-Schrödinger system can be derived as a diffusion limit of a Boltzmanntype model. In $\S 2$, we prove Theorem 1.2. The strategy of the proof as well as various notations are detailed in $\S 2.1$. Note that two essential ingredients are used: the first is a relative entropy inequality that provides preliminary estimates on the solution which are then completed with an $L^{2}$ estimate on the surface density. The second ingredient is the analysis of the Schrödinger-Poisson system (1.2), (1.3), which is shown to be uniquely solvable by convex minimization techniques in the spirit of [27-29]. Section 3 is devoted to the proof of Theorem 1.5, which uses a quadratic approximation of the relative entropy given in $\S 2$ and which is a Lyapunov functional for the linearized system around the stationary solution. The appendix is devoted to some technical lemmas and to classical results for Sturm-Liouville operators.

\subsection{Formal derivation from kinetic theory}

The drift-diffusion-Schrödinger system (1.2), (1.5), (1.6) can be derived as a diffusion limit of a kinetic system for partially quantized particles, called the kinetic subband system. More precisely, for a partially quantized system, the particle density can be written

$$
N(t, x, z)=\sum_{k=1}^{+\infty} \rho_{k}(t, x)\left|\chi_{k}(t, x, z)\right|^{2}
$$

where $\chi_{k}$ is given by $(1.2)$. In the physics terminology $[\mathbf{5}, \mathbf{1 2}, \mathbf{1 7}]$, the wave function $\chi_{k}$ is called the wave function of the $k$ th subband and $\epsilon_{k}$ its energy. The surface densities $\rho_{k}(t, x)$ are the occupation numbers of the subbands and are given in the kinetic framework by

$$
\rho_{k}(t, x)=\int_{\mathbb{R}^{2}} f_{k}(t, x, v) \mathrm{d} v
$$


where $f_{k}$ are solutions of kinetic equations in which the electrostatic potential energy $V$ is replaced by the subband energy $\epsilon_{k}$. In the collisionless case, such a model, which in quantum chemistry is related to the so-called Born-Oppenheimer approximation [22, $\mathbf{3 3}, \mathbf{3 4}]$, was obtained in [7] by a partial semi-classical limit of the Schrödinger equation and analysed in $[\mathbf{6}, \mathbf{8}]$. In order to obtain the diffusive regime, we introduce intersubband collisions $[\mathbf{1}, \mathbf{5}]$ in the Fermi golden-rule approximation

$$
\partial_{t} f_{k}^{\eta}+\frac{1}{\eta}\left\{\mathcal{H}_{k}, f_{k}^{\eta}\right\}=\frac{1}{\eta^{2}} Q\left(f^{\eta}\right)_{k}
$$

where $\eta$ is the scaled mean-free path assumed to be small and $\{\cdot, \cdot\}$ is the Poisson bracket $\{g, h\}=\nabla_{x} h \cdot \nabla_{v} g-\nabla_{v} h \cdot \nabla_{x} g$, Moreover, $\mathcal{H}_{p}$ is the energy of the system in the $k$ th subband:

$$
\mathcal{H}_{k}(t, x, v)=\frac{1}{2} v^{2}+\epsilon_{k}(t, x),
$$

where we recall that $\epsilon_{k}$ is the subband energy. The collision operator $Q$ is defined by

$$
Q(f)_{p}=\sum_{k^{\prime}} \int_{\mathbb{R}^{2}} \alpha_{k, k^{\prime}}\left(v, v^{\prime}\right)\left(\mathcal{M}_{k}(v) f_{k^{\prime}}\left(v^{\prime}\right)-\mathcal{M}_{k^{\prime}}\left(v^{\prime}\right) f_{k}(v)\right) \mathrm{d} v^{\prime},
$$

where $\alpha$ depends on the system and the function $\mathcal{M}_{k}$ is the Maxwellian:

$$
\mathcal{M}_{k}(t, x, v)=\frac{1}{2 \pi \mathcal{Z}} \mathrm{e}^{-\mathcal{H}_{k}(t, x, v)} .
$$

The diffusion limit consists in letting $\eta \rightarrow 0$ (a rigorous study of this limit will be the object of a future work). Admitting that $f_{k}^{\eta}$ converges towards a limit $f_{k}^{0}$ as $\eta \rightarrow 0$, we then have $f_{k}^{0} \in \operatorname{ker} Q$, which can be shown to be equal to

$$
\operatorname{ker} Q=\left\{f \text { such that } \exists \rho \in \mathbb{R}: f_{k}=\rho \mathcal{M}_{k}, \forall k \geqslant 1\right\} \text {. }
$$

Therefore,

$$
f_{k}^{0}(t, x, v)=N_{\mathrm{s}}(t, x) \mathcal{M}_{k}(t, x, v) .
$$

We remark that $N_{\mathrm{s}}(t, x)=\sum_{k} \int_{\mathbb{R}^{2}} f_{k}^{0}(t, x, v) \mathrm{d} v$ is the surface density of particles. Identifying the terms in (1.14) and letting $\eta \rightarrow 0$, one can prove, in the same spirit as in previous works on diffusion approximation $[\mathbf{2 1}, \mathbf{3 1}]$, that $N_{\mathrm{s}}$ satisfies the drift-diffusion equation

$$
\partial_{t} N_{\mathrm{s}}-\operatorname{div}_{x}\left(\mathbb{D}\left(\nabla_{x} N_{\mathrm{s}}+N_{\mathrm{s}} \nabla_{x} V_{\mathrm{s}}\right)\right)=0
$$

where $V_{\mathrm{s}}$ is the effective potential defined by (1.6) and $\mathbb{D}$ is a diffusion matrix (symmetric positive definite) depending on the choice of the transition rates $\alpha_{k, k^{\prime}}$. In this paper, we consider for simplicity the case $\mathbb{D}=\mathbb{I}$, where $\mathbb{I}$ is the identity matrix in $\mathbb{R}^{2}$.

\section{Existence and uniqueness (proof of Theorem 1.2)}

\subsection{Notations and strategy of the proof}

As in [6], we view the system as a two-dimensional drift-diffusion equation (1.5) for the surface density coupled to the quasi-static Schrödinger-Poisson system (1.2), (1.3). 
The drift-diffusion equation determines the value of the surface density in terms of the electrostatic potential, while the Schrödinger-Poisson systems allows us to compute the potential as a function of the surface density.

The overall problem is then solved by a fixed-point procedure for the unknown $N_{\mathrm{s}}$, as for the standard drift-diffusion-Poisson problem $[\mathbf{1 8 , 2 5 , 2 6}]$. The global-in-time existence relies heavily on an entropy estimate.

The first step now in the proof is to consider the quasi-static Schrodinger-Poisson system which consists, for any given non-negative function $N_{\mathrm{s}}(x)$ defined on $\omega$, in finding a potential $V(x, z)$ defined on $\Omega$ and satisfying

$$
\left.\begin{array}{c}
-\Delta_{x, z} V=N(x, z), \quad(x, z) \in \Omega ; \\
N(x, z)=N_{\mathrm{s}}(x) \sum_{k=1}^{+\infty} \frac{\mathrm{e}^{-\epsilon_{k}(x)}}{\mathcal{Z}(x)}\left|\chi_{k}(x, z)\right|^{2} ; \\
\mathcal{Z}(x)=\sum_{\ell=1}^{+\infty} \mathrm{e}^{-\epsilon_{\ell}(x)} \\
-\frac{1}{2} \partial_{z}^{2} \chi_{k}+V \chi_{k}=\epsilon_{k} \chi_{k}, \quad k \geqslant 1, \\
\chi_{k}(t, x, \cdot) \in H_{0}^{1}(0,1), \quad \int_{0}^{1} \chi_{k} \chi_{\ell} \mathrm{d} z=\delta_{k \ell},
\end{array}\right\}
$$

For this problem, we have the following result, whose proof is postponed.

Proposition 2.1. Let $N_{\mathrm{s}} \in L^{2}(\omega)$ such that $N_{\mathrm{s}} \geqslant 0$. Then the system (2.1) admits a unique solution $\left(V,\left(\epsilon_{k}, \chi_{k}\right)_{k \geqslant 1}\right)$, which satisfies the estimates $\|V\|_{H^{2}(\Omega)} \leqslant C\left(N_{\mathrm{s}}\right)$, the constant $C\left(N_{\mathrm{s}}\right)$ depending only on the $L^{2}(\omega)$ norm of $N_{\mathrm{s}}$. Moreover, for two arbitrary data $N_{\mathrm{s}}$ and $\tilde{N}_{\mathrm{s}}$, the corresponding solutions satisfy

$$
\|V-\tilde{V}\|_{H^{2}(\Omega)} \leqslant C\left(N_{\mathrm{s}}, \tilde{N}_{\mathrm{s}}\right)\left\|N_{\mathrm{s}}-\tilde{N}_{\mathrm{s}}\right\|_{L^{2}(\omega)} .
$$

In order to prove existence of solutions of the overall problem, we need to show some $a$ priori estimates for the solution. We shall begin with a relative entropy inequality (see, for example, $[\mathbf{2 , 3}, \mathbf{1 6}]$ for classical counterparts), then show a uniform $L^{p}$ estimate for the surface density. In order to do so, we proceed as in the standard drift-diffusion case [18] and define the slotboom variable

$$
u=\mathrm{e}^{\epsilon_{F}}=\frac{N_{\mathrm{s}}}{\mathcal{Z}}
$$

We also define the surface current density

$$
J_{\mathrm{s}}=-\nabla_{x} N_{\mathrm{s}}-N_{\mathrm{s}} \nabla_{x} V_{\mathrm{s}}=-\sum_{k} \mathrm{e}^{-\epsilon_{k}} \nabla_{x} u
$$

in such a way that the drift-diffusion equation may be written

$$
\partial_{t} N_{\mathrm{s}}+\operatorname{div}_{x} J_{\mathrm{s}}=0 .
$$


We denote by $\rho_{k}$ the occupation factor of the $k$ th subband:

$$
\rho_{k}=u \mathrm{e}^{-\epsilon_{k}}
$$

so that

$$
N=\sum_{k} \rho_{k}\left|\chi_{k}\right|^{2}, \quad N_{\mathrm{s}}=\sum_{k} \rho_{k} .
$$

Now we introduce two extensions $\underline{N_{\mathrm{s}}}$ and $\underline{V}$ of the boundary data. These extensions are respectively defined on $\omega$ and $\Omega$ and chosen in such a way that:

(i) $\underline{N_{\mathrm{s}}} \in C^{2}(\bar{\omega}), 0<\underline{N_{1}} \leqslant \underline{N_{\mathrm{s}}} \leqslant \underline{N_{2}}$ with two non-negative constants $N_{1}$ and $N_{2}$, and $\left.\underline{N_{\mathrm{s}}}\right|_{\partial \omega}=N_{b}$

(ii) $\underline{V} \in C^{2}(\bar{\Omega})$ and satisfies the boundary conditions $\left.\underline{V}\right|_{\partial \omega \times(0,1)}=V_{b}$ and $\partial_{z} \underline{V}(x, 0)=$ $\partial_{z} \underline{V}(x, 1)=0$, for all $x \in \omega$.

It is clear that for sufficiently regular domains such functions exist. Solving (1.2) with $\underline{V}$ instead of $V$, we find two sequences $\epsilon_{k}[\underline{V}](x)$ and $\chi_{k}[\underline{V}](x, z)$, which we shall shortly denote by $\underline{\epsilon_{k}}$ and $\underline{\chi_{k}}$. We then define $\underline{u}, \underline{\epsilon_{F}}, \underline{\mathcal{Z}}$ and $\underline{\rho_{k}}$ by

$$
\underline{u}=\frac{N_{\mathrm{s}}}{\underline{\mathcal{Z}}}, \quad \underline{\mathcal{Z}}=\sum_{k} \mathrm{e}^{-\underline{\epsilon_{k}}}, \quad \underline{\epsilon_{F}}=\log \underline{u} ; \quad \underline{\rho_{k}}=\underline{u} \mathrm{e}^{-\underline{\epsilon_{k}}}=\mathrm{e}^{\underline{\epsilon_{F}}-\underline{\epsilon_{k}}},
$$

as well as the density

$$
\underline{N}(x, z)=\sum_{k} \underline{\rho_{k}}(x)\left|\underline{\chi_{k}}(x, z)\right|^{2} .
$$

It may readily be seen that

$$
\left\|\frac{\nabla_{x} \underline{u}}{\underline{u}}\right\|_{L^{\infty}(\omega)}<\infty .
$$

The relative entropy of $\left(\rho_{k}, V\right)$ with respect to $\left(\underline{\rho_{k}}, \underline{V}\right)$ is defined by

$$
\begin{aligned}
W=\sum_{k} \int_{\omega}\left(\rho_{k} \log \left(\rho_{k} / \underline{\rho_{k}}\right)-\rho_{k}\right. & \left.+\underline{\rho_{k}}\right) \mathrm{d} x+\frac{1}{2} \iint_{\Omega}\left|\nabla_{x, z}(V-\underline{V})\right|^{2} \mathrm{~d} x \mathrm{~d} z \\
& +\int_{\omega} \sum_{k} u \mathrm{e}^{-\epsilon_{k}}\left(\epsilon_{k}[V]-\epsilon_{k}[\underline{V}]-\left\langle\left|\chi_{k}\right|^{2}(V-\underline{V})\right\rangle\right) \mathrm{d} x,
\end{aligned}
$$

where we use the notation $\langle f\rangle=\int_{0}^{1} f \mathrm{~d} z$. As will be shown later on, the three terms on the right-hand side of the above identity are non-negative. Besides, $W$ has the following compact form:

$$
W=\iint_{\Omega}\left(N\left(\epsilon_{F}-V-\left(\underline{\epsilon_{F}}-\underline{V}\right)\right)-N+\underline{N}\right) \mathrm{d} x \mathrm{~d} z+\frac{1}{2} \iint_{\Omega}\left|\nabla_{x, z}(V-\underline{V})\right|^{2} \mathrm{~d} x \mathrm{~d} z .
$$

Let us comment on this formula. One can note that the familiar form of the relative entropy for classical drift-diffusion systems is recovered here. The main difference is that, in the classical case, the relation between the Fermi level, the electrostatic potential and 
the density is local: $\epsilon_{F}-V=\log N$ (see, for example, $[\mathbf{2}, \mathbf{9}, \mathbf{1 8}]$ ), while here this relation is non-local in space. This form is also similar to the one recently obtained in $[\mathbf{2 0}]$ for a fully quantum drift-diffusion (QDD) model. This model was derived in [14] by following the strategy of quantum moments developed in $[\mathbf{1 3}]$ (see also the review paper $[\mathbf{1 5}]$ ). It consists of a three-dimensional drift-diffusion equation involving a quantum chemical potential that depends on the density in a non-local way, via the resolution of a quasistatic auxiliary quantum problem. In the QDD model, the quantum chemical potential is the generalization of the term $\epsilon_{F}-V$ of the present model.

The following two propositions provide some a priori estimates needed for the resolution of the coupled system.

Proposition 2.2. Let $T>0$. Let $\left(N_{\mathrm{s}}, V\right)$ be a weak solution of (1.2), (1.3), (1.5) and (1.11) such that $N_{\mathrm{s}} \in C\left([0, T], L^{2}(\omega)\right) \cap L^{2}\left([0, T], H^{1}(\omega)\right)$ and $V \in C\left([0, T], H^{2}(\Omega)\right)$. Then we have

$$
\forall t \in[0, T], \quad 0 \leqslant W(t)<C_{T},
$$

where $C_{T}$ is a constant only depending on $T, W(0)$ and $\underline{u}$.

Proposition 2.3. Let $T>0$ and assume that $N_{\mathrm{s}}^{0} \in L^{p}(\omega)$ for some $p \in[2,+\infty]$ and let $\left(N_{\mathrm{s}}, V\right)$ be weak solution of (1.5), (1.2), (1.3), (1.11) such that $N_{\mathrm{s}} \in C\left([0, T], L^{2}(\omega)\right) \cap$ $L^{2}\left([0, T], H^{1}(\omega)\right)$ and $V \in C\left([0, T], H^{2}(\Omega)\right)$. Then

$$
N_{\mathrm{s}} \in C\left([0, T], L^{p}(\omega)\right),
$$

for any $T>0$, with a bound depending only on $T, N_{b}, V_{b}$ and $\left\|N_{\mathrm{s}}^{0}\right\|_{L^{p}(\omega)}$.

\subsection{Proof of the entropy inequality}

The aim of this subsection is the proof of Proposition 2.2. Let $\left(N_{\mathrm{s}}, V\right)$ be a weak solution of (1.2), (1.3) and (1.5). Since $V \in C\left([0, T], H^{2}(\Omega)\right)$, by Lemma A 6, we deduce that $V_{\mathrm{s}} \in C\left([0, T], H^{2}(\Omega)\right)$. This is sufficient to ensure that $N_{\mathrm{s}} \geqslant 0$, due to the maximum principle for parabolic equations (see, for example, $[\mathbf{2 4}]$ ).

\subsubsection{The relative entropy is the sum of three positive terms}

Let us now show that the relative entropy $W$ defined by (2.6) is non-negative. This is obviously the case for the first two terms. In order to deal with the third term, let us define $\epsilon_{k}^{s}:=\epsilon_{k}[s V+(1-s) \underline{V}]$, and $\chi_{k}^{s}=\chi_{k}[s V+(1-s) \underline{V}]$. Straightforward computations using Lemma A 3 in the appendix lead to

$$
\begin{aligned}
\sum_{k} u \mathrm{e}^{-\epsilon_{k}}\left(\epsilon_{k}[V]\right. & \left.-\epsilon_{k}[\underline{V}]-\left\langle\left|\chi_{k}\right|^{2}(V-\underline{V})\right\rangle\right) \\
& =\int_{0}^{1} \int_{1}^{s} \sum_{k, \ell \neq k} u \frac{\mathrm{e}^{-\epsilon_{k}}-\mathrm{e}^{-\epsilon_{\ell}}}{\epsilon_{k}^{\sigma}-\epsilon_{\ell}^{\sigma}}\left\langle\chi_{k}^{\sigma}(V-\underline{V}) \chi_{\ell}^{\sigma}\right\rangle^{2} \mathrm{~d} \sigma \mathrm{d} s \\
& \geqslant 0,
\end{aligned}
$$

since the sequence $\left(\epsilon_{k}=\epsilon_{k}[V]\right)_{k \geqslant 1}$ is increasing. This is sufficient to conclude that $W \geqslant 0$, as the sum of three non-negative terms. 


\subsubsection{The initial relative entropy is finite}

Since $N_{\mathrm{s}}^{0} \in L^{2}(\omega)$, by Proposition 2.1 we have $V \in H^{2}(\Omega) \subset L^{\infty}(\Omega)$. From Lemma A 1 we deduce that

$$
\left\|\epsilon_{k}-\frac{1}{2} \pi^{2} k^{2}\right\|_{L^{\infty}(\omega)} \leqslant\|V\|_{L^{\infty}(\Omega)} .
$$

This is sufficient to deduce that $W(0)<+\infty$.

\subsubsection{Relative entropy dissipation}

Let us now compute $\mathrm{d} W / \mathrm{d} t$. We first remark that

$$
\frac{\mathrm{d}}{\mathrm{d} t} \sum_{k} \int_{\omega}\left(\rho_{k} \log \left(\frac{\rho_{k}}{\rho_{k}}\right)-\rho_{k}+\underline{\rho_{k}}\right) \mathrm{d} x=\sum_{k} \int_{\omega} \partial_{t} \rho_{k} \log \left(\frac{\rho_{k}}{\rho_{k}}\right) \mathrm{d} x .
$$

Taking advantage of the identity $N_{\mathrm{s}}=\sum \rho_{k}$ and from $\log \rho_{k}=\log u-\epsilon_{k}$, the right-hand side is equal to

$$
\int_{\omega} \partial_{t} N_{\mathrm{s}} \log \left(\frac{u}{\underline{u}}\right) \mathrm{d} x-\sum_{k} \int_{\omega} \partial_{t} \rho_{k}\left(\epsilon_{k}-\underline{\epsilon_{k}}\right) \mathrm{d} x .
$$

With the identity $\partial_{t} \epsilon_{k}=\left\langle\left|\chi_{k}\right|^{2} \partial_{t} V\right\rangle$ (see Lemma A 3) and (1.5) we obtain

$$
\begin{aligned}
\frac{\mathrm{d}}{\mathrm{d} t} \sum_{k} \int_{\omega}\left(\rho_{k} \log \left(\rho_{k} / \underline{\rho_{k}}\right)\right. & \left.-\rho_{k}+\underline{\rho_{k}}\right) \mathrm{d} x \\
= & \int_{\omega} \operatorname{div}_{x}\left(\sum_{k} \mathrm{e}^{-\epsilon_{k}} \nabla_{x} u\right) \log \frac{u}{\underline{u}} \mathrm{~d} x \\
& \quad-\frac{\mathrm{d}}{\mathrm{d} t} \int_{\omega} \sum_{k} \rho_{k}\left(\epsilon_{k}-\underline{\epsilon_{k}}\right) \mathrm{d} x+\iint_{\Omega} \sum_{k} \rho_{k}\left|\chi_{k}\right|^{2} \partial_{t} V \mathrm{~d} x \mathrm{~d} z .
\end{aligned}
$$

The Poisson equation and the fact that $V=\underline{V}$ on $\partial \omega \times(0,1)$ give

$$
\begin{aligned}
\frac{\mathrm{d}}{\mathrm{d} t} \frac{1}{2} \iint_{\Omega}\left|\nabla_{x, z}(V-\underline{V})\right|^{2} \mathrm{~d} x \mathrm{~d} z & =\iint_{\Omega} \partial_{t} N(V-\underline{V}) \mathrm{d} x \mathrm{~d} z \\
& =\frac{\mathrm{d}}{\mathrm{d} t} \iint_{\Omega} N(V-\underline{V}) \mathrm{d} x \mathrm{~d} z-\iint_{\Omega} N \partial_{t} V \mathrm{~d} x \mathrm{~d} z
\end{aligned}
$$

By using (1.6) and the expression of $\rho_{k}$, we obtain

$$
\frac{\mathrm{d}}{\mathrm{d} t} W=\int_{\omega} \operatorname{div}_{x}\left(\sum_{k} \mathrm{e}^{-\epsilon_{k}} \nabla_{x} u\right) \log \left(\frac{u}{\underline{u}}\right) \mathrm{d} x .
$$

After an integration by parts, owing to $u=\underline{u}$ on $\partial \omega$ we may deduce that

$$
\frac{\mathrm{d}}{\mathrm{d} t} W=-\int_{\omega} \sum_{k} \mathrm{e}^{-\epsilon_{k}} \frac{\left|\nabla_{x} u\right|^{2}}{u} \mathrm{~d} x+\int_{\omega} \sum_{k} \mathrm{e}^{-\epsilon_{k}} \frac{\nabla_{x} u \cdot \nabla_{x} \underline{u}}{\underline{u}} \mathrm{~d} x .
$$

In the following, we shall use the notation

$$
D(t)=\int_{\omega} \sum_{k} \mathrm{e}^{-\epsilon_{k}} \frac{\left|\nabla_{x} u\right|^{2}}{u} \mathrm{~d} x
$$


and shall refer to this term as the entropy dissipation rate. Let us now define $\beta=$ $\left\|\nabla_{x} \underline{u} / \underline{u}\right\|_{L^{\infty}(\omega)}<+\infty$ (from (2.5)). A straightforward Cauchy-Schwarz inequality leads to

$$
\frac{\mathrm{d}}{\mathrm{d} t} W+D \leqslant \beta \sqrt{D} \sqrt{\left\|N_{\mathrm{s}}\right\|_{L^{1}(\omega)}} .
$$

Using the inequality $2 a b \leqslant \varepsilon^{2} a^{2}+1 / \varepsilon^{2} b^{2}$ for $\varepsilon>0$ sufficiently small, we get

$$
\frac{\mathrm{d}}{\mathrm{d} t} W \leqslant C\left\|N_{\mathrm{s}}\right\|_{L^{1}(\omega)}
$$

Since the function $F(t)=t \log (t)-t+1$, satisfies $F(t) \geqslant t+(1-e)$, we obtain

$$
\begin{aligned}
W & \geqslant \sum_{k} \int_{\omega} \underline{\rho_{k}} F\left(\rho_{k} / \underline{\rho_{k}}\right) \mathrm{d} x \\
& \geqslant \sum_{k} \int_{\omega} \underline{\rho_{k}}\left(\rho_{k} / \underline{\rho_{k}}+1-\mathrm{e}\right) \mathrm{d} x \\
& \geqslant \int_{\omega} N_{\mathrm{s}} \mathrm{d} x-(e-1) \int_{\omega} \underline{N_{\mathrm{s}}} \mathrm{d} x,
\end{aligned}
$$

which leads to the differential inequality

$$
\frac{\mathrm{d}}{\mathrm{d} t} W \leqslant C \int_{\omega} N_{\mathrm{s}} \mathrm{d} x \leqslant C\left(W+C_{0}\right),
$$

where $C_{0}$ depends only on the data of the problem (and not on the considered solution). The Gronwall lemma implies that $W(t) \leqslant C_{T}$ for all $t \in[0, T]$, where $C_{T}$ depends only on $T, W(0)$ and data $\left(W(0)<+\infty\right.$ if $\left.N_{\mathrm{s}}^{0} \in L^{2}(\omega)\right)$.

Remark 2.4. The above manipulations are formal for weak solutions (defined such that $\left.N_{\mathrm{s}} \in C\left([0, T], L^{2}(\omega)\right)\right)$. To make the argument rigorous, it is sufficient to regularize the data, obtain a regular solution for which the result holds, then pass to the limit in the regularization parameter and use the uniqueness of the weak solution (proved in $\S 2.5$ ).

\subsection{Proof of the $L^{p}$ estimate}

The aim of this subsection is the proof of Proposition 2.3. We have seen in $\S 2.2$ that $W(0)<C\left(\left\|N_{\mathrm{s}}^{0}\right\|_{L^{2}(\omega)}\right)$. Hence, Proposition 2.2 implies that

$$
\forall t \leqslant T, \quad\|V(t)\|_{H^{1}(\Omega)}+\left\|N_{\mathrm{s}}(t)\right\|_{L^{1}(\omega)} \leqslant C_{T} .
$$

Due to the Trudinger inequality (A 8) and to (A 6), as well as Lemma A 5 the functions

$$
S_{1}(t, x)=\sup _{k \geqslant 1}\left\|\chi_{k}(t, x, \cdot)\right\|_{L_{z}^{\infty}}^{2}, \quad S_{2}(t, x)=\sum_{k \geqslant 1} \frac{\mathrm{e}^{-\epsilon_{k}(t, x)}}{\mathcal{Z}(t, x)}\left(\epsilon_{k}(t, x)\right)^{2}
$$

are in $L^{\infty}\left((0, T), L^{p}(\omega)\right)$ for any finite $p$ and satisfy the bound

$$
\forall p<+\infty, \quad\left\|S_{1}(t, \cdot)\right\|_{L^{p}(\omega)}+\left\|S_{2}(t, \cdot)\right\|_{L^{p}(\omega)} \leqslant C_{p},
$$

where $C_{p}$ is a constant depending only on $\|V(t)\|_{H^{1}(\Omega)}$. From now on, we define

$$
n_{\mathrm{s}}=N_{\mathrm{s}}-\underline{N_{\mathrm{s}}}, \quad n=N-\underline{N}, \quad v_{\mathrm{s}}=V_{\mathrm{s}}-\underline{V_{\mathrm{s}}}, \quad v=V-\underline{V} .
$$




\subsubsection{Proof of Proposition 2.3 for $p \in[2,+\infty)$}

Multiply (1.5) by $n_{\mathrm{s}}\left|n_{\mathrm{s}}\right|^{p-2}$ and integrate on $\omega$. After an integration by parts, we get

$$
\begin{aligned}
\frac{1}{p} \frac{\mathrm{d}}{\mathrm{d} t} \int_{\omega}\left|n_{\mathrm{s}}\right|^{p} \mathrm{~d} x+(p-1) \int_{\omega}\left|\nabla_{x} n_{\mathrm{s}}\right|^{2}\left|n_{\mathrm{s}}\right|^{p-2} \mathrm{~d} x+\frac{p-1}{p} \int_{\omega} \nabla_{x}\left|n_{\mathrm{s}}\right|^{p} \cdot \nabla_{x} V_{\mathrm{s}} \mathrm{d} x \\
=\int_{\omega} \Delta_{x} \underline{N_{\mathrm{s}}} n_{\mathrm{s}}\left|n_{\mathrm{s}}\right|^{p-2} \mathrm{~d} x+\int_{\omega} \operatorname{div}_{x}\left(\underline{N_{\mathrm{s}}} \nabla_{x} V_{\mathrm{s}}\right) n_{\mathrm{s}}\left|n_{\mathrm{s}}\right|^{p-2} \mathrm{~d} x .
\end{aligned}
$$

After an integration by parts, the last term on the left-hand side can be written as

$$
-\frac{p-1}{p} \int_{\omega}\left|n_{\mathrm{s}}\right|^{p} \Delta_{x} V_{\mathrm{s}} \mathrm{d} x \text {. }
$$

The above computations follow closely the standard drift-diffusion Poisson system for which the above term is non-negative. In our case, however, $-\Delta_{x} V_{\mathrm{s}} \neq N_{\mathrm{s}}$, which induces additional difficulties. Indeed, with the Poisson equation (1.3), we have $-\Delta_{x} V=\partial_{z}^{2} V+N$. And, after some integrations by parts,

$$
\left\langle\partial_{z}^{2} V\left|\chi_{k}\right|^{2}\right\rangle=2\left\langle V \chi_{k} \partial_{z}^{2} \chi_{k}\right\rangle+2\left\langle V\left|\partial_{z} \chi_{k}\right|^{2}\right\rangle
$$

From the Schrödinger equation (1.2), we have

$$
\partial_{z}^{2} \chi_{k}=2\left(V-\epsilon_{k}\right) \chi_{k} \quad \text { and } \quad 2\left\langle V\left|\chi_{k}\right|^{2}\right\rangle+\left|\partial_{z} \chi_{k}\right|^{2}=2 \epsilon_{k} .
$$

Thus,

$$
\left\langle\partial_{z}^{2} V\left|\chi_{k}\right|^{2}\right\rangle=4\left\langle V^{2}\left|\chi_{k}\right|^{2}\right\rangle+2\left\langle\left(V+\epsilon_{k}\right)\left|\partial_{z} \chi_{k}\right|^{2}\right\rangle-4 \epsilon_{k}^{2} .
$$

These remarks lead to the following identity:

$$
\begin{aligned}
-\Delta_{x} V_{\mathrm{s}}=- & 4 S_{2}(t, x)+\frac{\left\langle N^{2}+4 V^{2} N\right\rangle}{N_{\mathrm{s}}}+2 \sum_{k} \frac{\mathrm{e}^{-\epsilon_{k}}}{\mathcal{Z}}\left\langle\left(V+\epsilon_{k}\right)\left|\partial_{z} \chi_{k}\right|^{2}\right\rangle \\
& -\frac{1}{\mathcal{Z}} \sum_{k} \sum_{\ell \neq k}\left(\frac{\mathrm{e}^{-\epsilon_{k}}-\mathrm{e}^{-\epsilon_{\ell}}}{\epsilon_{k}-\epsilon_{\ell}}\right)\left\langle\chi_{k} \chi_{\ell} \nabla_{x} V\right\rangle^{2} \\
& +\sum_{k} \frac{\mathrm{e}^{-\epsilon_{k}}}{\mathcal{Z}}\left\langle\left|\chi_{k}\right|^{2} \nabla_{x} V\right\rangle^{2}-\left(\sum_{k} \frac{\mathrm{e}^{-\epsilon_{k}}}{\mathcal{Z}}\left\langle\left|\chi_{k}\right|^{2} \nabla_{x} V\right\rangle\right)^{2},
\end{aligned}
$$

where $S_{2}$ is defined in (2.10). By the Cauchy-Schwarz inequality, the sum of the last two terms on the right-hand side is non-negative. Moreover, except for the first one, the other terms are obviously non-negative. By an integration by parts, we deduce that

$$
\frac{1}{p} \frac{\mathrm{d}}{\mathrm{d} t} \int_{\omega}\left|n_{\mathrm{s}}\right|^{p} \mathrm{~d} x+(p-1) \int_{\omega}\left|\nabla_{x} n_{\mathrm{s}}\right|^{2}\left|n_{\mathrm{s}}\right|^{p-2} \mathrm{~d} x \leqslant \mathrm{I}+\mathrm{II}+\mathrm{III},
$$

where

$$
\begin{aligned}
\mathrm{I} & =4 \frac{p-1}{p} \int_{\omega}\left|n_{\mathrm{s}}\right|^{p} S_{2} \mathrm{~d} x, \\
\mathrm{II} & =\int_{\omega} \Delta_{x} \underline{N_{\mathrm{s}}} n_{\mathrm{s}}\left|n_{\mathrm{s}}\right|^{p-2} \mathrm{~d} x, \\
\mathrm{III} & =\int_{\omega} \operatorname{div}_{x}\left(\underline{N_{\mathrm{s}}} \nabla_{x} V_{\mathrm{s}}\right) n_{\mathrm{s}}\left|n_{\mathrm{s}}\right|^{p-2} \mathrm{~d} x .
\end{aligned}
$$


Let us now analyse each term separately.

First we estimate I. Thanks to the Hölder inequality, for all $r>1$ and $r^{\prime}=r /(r-1)$ we have

$$
\mathrm{I}=4 \frac{p-1}{p} \int_{\omega}\left|n_{\mathrm{s}}\right|^{p} S_{2} \mathrm{~d} x \leqslant C\left\|\left|n_{\mathrm{s}}\right|^{p / 2}\right\|_{L^{2 r}}^{2}\left\|S_{2}\right\|_{L^{r^{\prime}}} .
$$

By applying Gagliardo-Nirenberg and Young inequalities, for $r>1$ we have

$$
\begin{aligned}
\left\|\left|n_{\mathrm{s}}\right|^{p / 2}\right\|_{L^{2 r}(\omega)}^{2} & \leqslant C\left\|\left|n_{\mathrm{s}}\right|^{p / 2}\right\|_{L^{2}(\omega)}^{2 / r}\left\|\left|n_{\mathrm{s}}\right|^{p / 2}\right\|_{H^{1}(\omega)}^{2(1-1 / r)} \\
& \leqslant C\left(\frac{1}{\varepsilon^{r}}\left\|n_{\mathrm{s}}\right\|_{L^{p}}^{p}+\varepsilon^{r /(r-1)}\left\|\left|n_{\mathrm{s}}\right|^{p / 2}\right\|_{H^{1}(\omega)}^{2}\right) .
\end{aligned}
$$

By using the estimate (2.11) and the Poincaré inequality we obtain

$$
\mathrm{I} \leqslant C_{\varepsilon}\left\|n_{\mathrm{s}}\right\|_{L^{p}}^{p}+\left.\left.C \varepsilon \int_{\omega}\left|\nabla_{x}\right| n_{\mathrm{s}}\right|^{p / 2}\right|^{2} \mathrm{~d} x .
$$

We now estimate II. This is an easy task. By a straightforward Hölder inequality, we have

$$
|\mathrm{II}|=\left.\left|\int_{\omega} \Delta_{x} \underline{N_{\mathrm{s}}} n_{\mathrm{s}}\right| n_{\mathrm{s}}\right|^{p-2} \mathrm{~d} x \mid \leqslant\left\|n_{\mathrm{s}}\right\|_{L^{p}(\omega)}^{p-1}\left\|\Delta_{x} \underline{N_{\mathrm{s}}}\right\|_{L^{p}(\omega)} .
$$

Estimating III requires more work. We first begin by an integration by parts and obtain

$$
\mathrm{III}=-(p-1) \iint_{\Omega} \frac{N_{\mathrm{s}}}{\sum_{k}} \frac{\mathrm{e}^{-\epsilon_{k}}}{\mathcal{Z}}\left|\chi_{k}\right|^{2} \nabla_{x} V \cdot \nabla_{x} n_{\mathrm{s}}\left|n_{\mathrm{s}}\right|^{p-2} \mathrm{~d} x \mathrm{~d} z
$$

This leads to the inequality

$$
|\mathrm{III}| \leqslant(p-1)\left\|\underline{N_{\mathrm{s}}}\right\|_{L^{\infty}} \iint_{\Omega}\left|S_{1}(t, x)\right|\left|\nabla_{x} V\right|\left|\nabla_{x} n_{\mathrm{s}}\right|\left|n_{\mathrm{s}}\right|^{p-2} \mathrm{~d} x \mathrm{~d} z,
$$

where $S_{1}$ is defined in (2.10). Taking advantage of (2.11), we find after a Hölder inequality that

$$
|\mathrm{III}| \leqslant C_{q, r}\left\|\underline{N_{\mathrm{S}}}\right\|_{L^{\infty}}\left\|\nabla_{x} V\right\|_{L^{q}}\left\|\nabla_{x} n_{\mathrm{S}}\left|n_{\mathrm{S}}\right|^{p-2}\right\|_{L^{r}}
$$

for any $(q, r)$ such that $q<+\infty$ and $r>q^{\prime}$, where $q^{\prime}=q /(q-1)$. By choosing $r=p / p-1$, by a Hölder inequality we have

$$
\left\|\nabla_{x} n_{\mathrm{s}}\left|n_{\mathrm{S}}\right|^{p-2}\right\|_{L^{r}} \leqslant\left\|\nabla_{x} n_{\mathrm{s}}\left|n_{\mathrm{S}}\right|^{(p-2) / 2}\right\|_{L^{2}}\left\|n_{\mathrm{s}}\right\|_{L^{p}}^{(p-2) / 2} .
$$

Now one can apply (2.17) with any $q>p$. By choosing $q$ close enough to $p$, the Sobolev inequality

$$
\left\|\nabla_{x} V\right\|_{L^{q}} \leqslant C_{1}\|V\|_{W^{2, s}} \leqslant C_{2}\|N\|_{L^{s}}+C_{3}
$$

holds for some $s<p$. Again by using the inequality

$$
N \leqslant N_{\mathrm{s}} S_{1}
$$


where $S_{1}$ is defined by (2.10) and satisfies the uniform bound (2.11), we immediately obtain $\|N\|_{L^{s}} \leqslant C\left\|N_{\mathrm{s}}\right\|_{L^{p}} \leqslant C\left(\left\|\underline{N_{\mathrm{S}}}\right\|_{L^{p}}+\left\|n_{\mathrm{s}}\right\|_{L^{p}}\right)$. Besides, we have

$$
\int_{\omega}\left|\nabla_{x} n_{\mathrm{s}}\right|^{2}\left|n_{\mathrm{s}}\right|^{p-2} \mathrm{~d} x=\frac{4}{p^{2}} \int_{\omega}\left|\nabla_{x}\left(\left|n_{\mathrm{s}}\right|^{p / 2}\right)\right|^{2} \mathrm{~d} x .
$$

All in all, (2.17) becomes

$$
|\mathrm{III}| \leqslant C\left\|\nabla_{x}\left(\left|n_{\mathrm{s}}\right|^{p / 2}\right)\right\|_{L^{2}(\omega)}\left\|n_{\mathrm{s}}\right\|_{L^{p}(\omega)}^{(p-2) / 2}\left(\left\|n_{\mathrm{s}}\right\|_{L^{p}(\omega)}+\left\|\underline{N_{\mathrm{s}}}\right\|_{L^{p}(\omega)}+1\right),
$$

which leads, after a Young inequality, to

$$
|\mathrm{III}| \leqslant C_{1} \varepsilon^{2} \int_{\omega}\left|\nabla_{x}\left(\left|n_{\mathrm{s}}\right|^{p / 2}\right)\right|^{2} \mathrm{~d} x+\frac{C_{2}}{\varepsilon^{2}}\left\|n_{\mathrm{s}}\right\|_{L^{p}(\omega)}^{p}+\frac{C_{3}}{\varepsilon^{2}}\left\|n_{\mathrm{s}}\right\|_{L^{p}(\omega)}^{p-2},
$$

where $\varepsilon$ is an arbitrarily small constant and $C_{1}, C_{2}$ and $C_{3}$ are independent of $\varepsilon$.

Now consider the inequality (2.14). Inserting the inequalities (2.15), (2.16), (2.19) and (2.20) in (2.14) and fixing $\varepsilon$ sufficiently small, there exist $A>0$ and non-negative constants still denoted by $C_{1}, C_{2}$ and $C_{3}$ such that

$$
\frac{1}{p} \frac{\mathrm{d}}{\mathrm{d} t} \int_{\omega}\left|n_{\mathrm{s}}\right|^{p} \mathrm{~d} x+A \int_{\omega}\left|\nabla_{x}\left(\left|n_{\mathrm{s}}\right|^{p / 2}\right)\right|^{2} \mathrm{~d} x \leqslant C_{1} \int_{\omega}\left|n_{\mathrm{s}}\right|^{p} \mathrm{~d} x+C_{2}\left\|n_{\mathrm{s}}\right\|_{L^{p}(\omega)}^{p-1}+C_{3}\left\|n_{\mathrm{s}}\right\|_{L^{p}(\omega)}^{p-2} .
$$

A Gronwall argument leads to the boundedness on $[0, T]$ of $\left\|n_{\mathbf{S}}(t)\right\|_{L^{p}(\omega)}$.

\subsubsection{Proof of Proposition 2.3 for $p=+\infty$}

Since $N_{\mathrm{s}} \in L^{p}(\omega)$ for all $1 \leqslant p<+\infty$, by (2.18) and (2.11), $n \in L^{r}(\Omega)$ for all $1 \leqslant r<+\infty$. Therefore, the Poisson equation (1.3) leads to $V \in W^{2, r}(\Omega)$. By Sobolev embeddings, the potential $V$ lies in $L^{\infty}([0, T] \times \Omega)$. Hence, from (2.13) and (A 2) we deduce that there exists a non-negative constant $a$ such that $\Delta_{x} V_{\mathrm{s}} \leqslant a$. We use the standard notation $f_{+}$for the positive part of $f$ :

$$
f_{+}= \begin{cases}f & \text { if } f \geqslant 0 \\ 0 & \text { otherwise. }\end{cases}
$$

Let us define

$$
A(t)=\lambda \mathrm{e}^{a t}, \quad \text { where } \lambda \geqslant \max \left(\left\|N_{\mathrm{s}}^{0}\right\|_{L^{\infty}(\omega)},\left\|N_{b}\right\|_{L^{\infty}(\partial \omega)}\right) .
$$

Then, from (1.5) and the choice of $a$,

$$
\partial_{t}\left(N_{\mathrm{s}}-A(t)\right)-\operatorname{div}_{x}\left(\nabla_{x}\left(N_{\mathrm{s}}-A(t)\right)+\left(N_{\mathrm{s}}-A(t)\right) \nabla_{x} V_{\mathrm{s}}\right) \leqslant 0 .
$$

Multiplying this equation by $\left(N_{\mathrm{s}}-A(t)\right)_{+}$and integrating over $\omega$ gives, after an integration by parts,

$$
\frac{1}{2} \frac{\mathrm{d}}{\mathrm{d} t} \int_{\omega}\left(N_{\mathrm{s}}-A(t)\right)_{+}^{2} \mathrm{~d} x+\int_{\omega}\left|\nabla_{x}\left(N_{\mathrm{s}}-A(t)\right)_{+}\right|^{2} \mathrm{~d} x+\frac{1}{2} \int_{\omega} \nabla_{x}\left(N_{\mathrm{s}}-A(t)\right)_{+}^{2} \nabla_{x} V_{\mathrm{s}} \mathrm{d} x \leqslant 0 .
$$


After another integration by parts, and since $\Delta_{x} V_{\mathrm{s}} \leqslant a$,

$$
\frac{\mathrm{d}}{\mathrm{d} t} \int_{\omega}\left(N_{\mathrm{s}}-A(t)\right)_{+}^{2} \mathrm{~d} x-a \int_{\omega}\left(N_{\mathrm{s}}-A(t)\right)_{+}^{2} \mathrm{~d} x \leqslant 0
$$

We deduce from this inequality and the choice of $\lambda$ in (2.21) that for all $t \in[0, T]$, $N_{\mathrm{s}} \leqslant A(t)$ and thus $N_{\mathrm{s}} \leqslant \lambda \mathrm{e}^{a T}$.

\subsection{Analysis of the Schrödinger-Poisson system}

In this subsection, we prove Proposition 2.1. We use the functional spaces

$$
H_{\omega}^{1}=\left\{V \in H^{1}(\Omega): \forall x \in \partial \omega, z \in[0,1], V(x, z)=0\right\}
$$

and

$$
L_{x}^{p} L_{z}^{q}(\Omega)=\left\{u \in L_{\mathrm{loc}}^{1}(\Omega) \text { such that }\|u\|_{L_{x}^{p} L_{z}^{q}(\Omega)}=\left(\int_{\omega}\|u(x, \cdot)\|_{L^{q}(0,1)}^{p} \mathrm{~d} x\right)^{1 / p}<+\infty\right\} .
$$

Due to Gagliardo-Nirenberg inequalities and interpolation estimates, one can prove the following lemma.

Lemma 2.5. We have the Sobolev embedding of $H^{1}(\Omega)$ into $L_{x}^{2} L_{z}^{\infty}(\Omega)$.

Let $V_{0} \in H^{2}(\Omega)$ be such that $V_{0}=V_{b}$ on $\partial \omega \times(0,1)$ and $\partial_{z} V_{0}(x, 0)=\partial_{z} V_{0}(x, 1)=0$ for all $x \in \omega$ (for instance, we can take $V_{0}=\underline{V}$ ). Proceeding as in $[\mathbf{6}]$ and in the spirit of $[\mathbf{2 7}]$, we can show that a weak solution of $(2.1)$ in the affine space $V_{0}+H_{\omega}^{1}$ is a critical point with respect to $V$ of the functional

$$
\begin{aligned}
J\left(V, N_{\mathrm{s}}\right) & =J_{0}(V)+J_{1}\left(V, N_{\mathrm{s}}\right) \\
& =\frac{1}{2} \iint_{\Omega}\left|\nabla_{x, z} V\right|^{2}+\int_{\omega} N_{\mathrm{s}} \log \sum_{k} \mathrm{e}^{-\epsilon_{k}[V]} \mathrm{d} x,
\end{aligned}
$$

where we recall that the $\left(\epsilon_{k}[V]\right)_{k \geqslant 1}$ denote the eigenvalues of the Hamiltonian

$$
-\frac{1}{2} \frac{\mathrm{d}^{2}}{\mathrm{~d} z^{2}}+V
$$

i.e. they satisfy (1.2).

The functional $J_{0}$ is clearly continuous and strongly convex on $H^{1}(\Omega)$. The analysis of the functional $V \mapsto J_{1}\left(V, N_{\mathrm{s}}\right)$ relies on the properties of $\epsilon_{k}[V]$. From the inequalities (see Lemma A 1)

$$
\left|\epsilon_{k}[V]-\epsilon_{k}[\tilde{V}]\right|(x) \leqslant\|V(x, \cdot)-\tilde{V}(x, \cdot)\|_{L_{z}^{\infty}(0,1)}
$$

and

$$
\log \frac{\sum_{k} \mathrm{e}^{-\epsilon_{k}[V]}}{\sum_{k} \mathrm{e}^{-\epsilon_{k}[\tilde{V}]}} \leqslant \log \frac{\sum_{k} \mathrm{e}^{-\epsilon_{k}[\tilde{V}]+\sup _{\ell}\left(\left|\epsilon_{\ell}[V]-\epsilon_{\ell}[\tilde{V}]\right|\right)}}{\sum_{k} \mathrm{e}^{-\epsilon_{k}[\tilde{V}]}}
$$


we deduce that

$$
\begin{aligned}
\left|J_{1}\left(V, N_{\mathrm{s}}\right)-J_{1}\left(\tilde{V}, N_{\mathrm{s}}\right)\right| & \leqslant \int_{\omega}\left|N_{\mathrm{s}}(x)\right| \sup _{k}\left(\left|\epsilon_{k}[V]-\epsilon_{k}[\tilde{V}]\right|(x)\right) \mathrm{d} x \\
& \leqslant\left\|N_{\mathrm{s}}\right\|_{L^{2}(\omega)}\|V-\tilde{V}\|_{L_{x}^{2} L_{z}^{\infty}(\Omega)} .
\end{aligned}
$$

The functional $J_{1}\left(\cdot, N_{\mathrm{s}}\right)$ is globally Lipschitz continuous on $L_{x}^{2} L_{z}^{\infty}(\Omega)$, and thus on $H^{1}(\Omega)$, due to Lemma 2.5.

Next, $J_{1}\left(\cdot, N_{\mathrm{s}}\right)$ is twice Gâteaux differentiable on $L^{\infty}(\Omega)$ and

$$
\begin{aligned}
\mathrm{d}_{V}^{2} J_{1}\left(V, N_{\mathrm{s}}\right) W \cdot W=- & \int_{\omega} \frac{N_{\mathrm{s}}}{\mathcal{Z}} \sum_{k} \sum_{\ell \neq k} \frac{\mathrm{e}^{-\epsilon_{k}}-\mathrm{e}^{-\epsilon_{\ell}}}{\epsilon_{k}-\epsilon_{\ell}}\left\langle\chi_{k} \chi_{\ell} W\right\rangle^{2} \mathrm{~d} x \\
& +\int_{\omega} N_{\mathrm{s}}\left\{\sum_{k} \frac{\mathrm{e}^{-\epsilon_{k}}}{\mathcal{Z}}\left\langle\left|\chi_{k}\right|^{2} W\right\rangle^{2}-\left(\frac{\sum_{k} \mathrm{e}^{-\epsilon_{k}}\left\langle\left|\chi_{k}\right|^{2} W\right\rangle}{\mathcal{Z}}\right)^{2}\right\} \mathrm{d} x .
\end{aligned}
$$

When $N_{\mathrm{s}}$ is non-negative, this quantity is non-negative thanks to the Cauchy-Schwarz inequality. Thus, $J_{1}\left(\cdot, N_{\mathrm{s}}\right)$ is convex. As a consequence, the functional $J\left(\cdot, N_{\mathrm{s}}\right)=J_{0}+$ $J_{1}\left(\cdot, N_{\mathrm{s}}\right)$ is continuous and strongly convex on $V_{0}+H_{\omega}^{1}$. Moreover, using the Poincaré inequality on $H_{\omega}^{1}$ and (2.22) with $\tilde{V}=0$, we have

$$
J\left(V, N_{\mathrm{s}}\right) \geqslant C\|V\|_{H^{1}(\Omega)}^{2}-C\left\|N_{\mathrm{s}}\right\|_{L^{2}(\Omega)}\|V\|_{H^{1}(\Omega)}+J\left(0, N_{\mathrm{s}}\right),
$$

and thus $J\left(\cdot, N_{\mathrm{s}}\right)$ is coercive and bounded from below on $H_{\omega}^{1}$ : it admits a unique minimizer, denoted by $V$, which is then the solution of our problem with the boundary conditions (1.11).

We now prove the $H^{2}$ estimate of $V$. Since $V$ is a minimizer of $J\left(\cdot, N_{\mathrm{s}}\right)$ we have $J\left(V, N_{\mathrm{s}}\right) \leqslant J\left(0, N_{\mathrm{s}}\right)$. Thus,

$$
\frac{1}{2} \iint_{\Omega}\left|\nabla_{x, z} V\right|^{2} \mathrm{~d} x \mathrm{~d} z \leqslant J_{1}\left(0, N_{\mathrm{s}}\right)-J_{1}\left(V, N_{\mathrm{s}}\right)
$$

Applying (2.22), we deduce that $V$ is bounded in $H^{1}(\Omega)$, with a bound only depending on the $L^{2}$ norm of $N_{\mathrm{s}}$. Therefore, the function $S_{1}$ defined in (2.10) satisfies the bound (2.11). Since $N \leqslant N_{\mathrm{s}} S_{1}$, we deduce that the density $N$ lies in $L^{r}(\Omega)$ for any $r<2$, which implies by elliptic regularity that $V \in W^{2, r}(\Omega)$. This implies that $V$ actually lies in $L^{\infty}$ which leads, in view of (A 6), to $S_{1} \in L^{\infty}$. Therefore, $N$ is bounded in $L^{2}(\Omega)$, which gives $V \in H^{2}(\Omega)$ by the elliptic regularity.

Let us now prove the Lipschitz dependence of $V$ with respect to $N_{\mathrm{s}}$ in $H^{2}(\Omega)$. Let $V$ and $\tilde{V}$ denote the minimizers of $J\left(\cdot, N_{\mathrm{s}}\right)$ and $J\left(\cdot, \tilde{N}_{\mathrm{s}}\right)$, respectively. Using the linearity of $J_{1}$ with respect to $N_{\mathrm{s}}$, its Lipschitz dependence with respect to $V$ from (2.22), the strong convexity of $J$ and the fact that $\tilde{V}$ minimizes $J\left(\cdot, \tilde{N}_{\mathrm{s}}\right)$, we get

$$
\begin{aligned}
\frac{1}{C}\|V-\tilde{V}\|_{H^{1}(\Omega)}^{2} & \leqslant J\left(\tilde{V}, N_{\mathrm{s}}\right)-J\left(V, N_{\mathrm{s}}\right) \\
& =J_{1}\left(\tilde{V}, N_{\mathrm{s}}-\tilde{N}_{\mathrm{s}}\right)-J_{1}\left(V, N_{\mathrm{s}}-\tilde{N}_{\mathrm{s}}\right)+J\left(\tilde{V}, \tilde{N}_{\mathrm{s}}\right)-J\left(V, \tilde{N}_{\mathrm{s}}\right) \\
& \leqslant C^{\prime}\|V-\tilde{V}\|_{H^{1}(\Omega)}\left\|N_{\mathrm{s}}-\tilde{N}_{\mathrm{s}}\right\|_{L^{2}(\omega)} .
\end{aligned}
$$


Thus, we have first the Lipschitz dependence of $V$ in $H^{1}(\Omega)$. The Poisson equation gives $-\Delta(V-\tilde{V})=N-\tilde{N}$, and

$$
\begin{aligned}
N-\tilde{N}=\left(N_{\mathrm{s}}-\tilde{N}_{\mathrm{s}}\right) \sum_{k} \frac{\mathrm{e}^{-\epsilon_{k}}\left|\chi_{k}\right|^{2}}{\mathcal{Z}} & \\
& +\tilde{N}_{\mathrm{s}} \sum_{k}\left(\frac{\mathrm{e}^{-\epsilon_{k}}}{\mathcal{Z}}-\frac{\mathrm{e}^{-\tilde{\epsilon}_{k}}}{\tilde{\mathcal{Z}}}\right)\left|\chi_{k}\right|^{2}+\tilde{N}_{\mathrm{s}} \sum_{k} \frac{\mathrm{e}^{-\tilde{\epsilon}_{k}}}{\tilde{\mathcal{Z}}}\left(\left|\chi_{k}\right|^{2}-\left|\tilde{\chi}_{k}^{2}\right|\right)
\end{aligned}
$$

(we use $\widetilde{\epsilon_{k}}$ as shorthand for $\epsilon_{k}[\tilde{V}]$ and $\tilde{\chi}_{k}$ for $\chi_{k}[\tilde{V}]$ ). With Lemma A 4,

$$
\left\|\chi_{k}-\tilde{\chi}_{k}\right\|_{L_{z}^{\infty}} \leqslant C_{1} \mathrm{e}^{C_{2}\left(\|V\|_{L_{z}^{2}}+\|\tilde{V}\|_{\left.L_{z}^{2}\right)}\right)}\|V-\tilde{V}\|_{L_{z}^{1}} .
$$

Denoting $\chi_{k}^{s}=\chi_{k}[\tilde{V}+s(V-\tilde{V})]$ and $\epsilon_{k}^{s}=\epsilon_{k}[\tilde{V}+s(V-\tilde{V})]$, we have with Lemma A 3,

$$
\begin{aligned}
\sum_{k}\left(\frac{\mathrm{e}^{-\epsilon_{k}}}{\mathcal{Z}}-\frac{\mathrm{e}^{-\tilde{\epsilon}_{k}}}{\tilde{\mathcal{Z}}}\right)\left|\chi_{k}\right|^{2}=\int_{0}^{1} \frac{\sum_{k}\left\langle\left|\chi_{k}^{s}\right|^{2}(V-\tilde{V})\right\rangle \mathrm{e}^{-\epsilon_{k}^{s}}}{\sum_{\ell} \mathrm{e}^{-\epsilon_{\ell}^{s}}} \frac{\sum_{k}\left|\chi_{k}\right|^{2} \mathrm{e}^{-\epsilon_{k}^{s}}}{\sum_{\ell} \mathrm{e}^{-\epsilon_{\ell}^{s}}} \mathrm{~d} s \\
-\int_{0}^{1} \sum_{k} \frac{\left\langle\left|\chi_{k}^{s}\right|^{2}(V-\tilde{V})\right\rangle \mathrm{e}^{-\epsilon_{k}^{s}}}{\sum_{\ell} \mathrm{e}^{-\epsilon_{\ell}^{s}}}\left|\chi_{k}\right|^{2} \mathrm{~d} s .
\end{aligned}
$$

Thus, since we have proved that $\chi_{k}^{s} \in L^{\infty}(\Omega)$, for all $s \in[0,1]$, we deduce that

$$
\left.\left|\sum_{k}\left(\frac{\mathrm{e}^{-\epsilon_{k}}}{\mathcal{Z}}-\frac{\mathrm{e}^{-\tilde{\epsilon}_{k}}}{\tilde{\mathcal{Z}}}\right)\right| \chi_{k}\right|^{2} \mid \leqslant C\|V-\tilde{V}\|_{L_{z}^{1}} .
$$

Hence, from (2.23) and (2.24), we deduce

$$
\|N-\tilde{N}\|_{L^{2}(\Omega)} \leqslant C\left\|N_{\mathrm{s}}-\tilde{N}_{\mathrm{s}}\right\|_{L^{2}(\omega)}+C\|V-\tilde{V}\|_{L^{2}(\Omega)} .
$$

Finally, from the Lipschitz dependence of $V$ with respect to $N_{\mathrm{s}}$ in $H^{1}(\Omega)$, we have locally

$$
\|V-\tilde{V}\|_{L^{2}(\Omega)} \leqslant C\left\|N_{\mathrm{s}}-\tilde{N}_{\mathrm{s}}\right\|_{L^{2}(\omega)} .
$$

Thus, $\|N-\tilde{N}\|_{L^{2}(\Omega)} \leqslant C\left\|N_{\mathrm{s}}-\tilde{N}_{\mathrm{s}}\right\|_{L^{2}(\omega)}$ with a constant $C$ depending on $\left\|N_{\mathrm{s}}\right\|_{L^{2}(\omega)}$ and $\left\|\tilde{N}_{\mathrm{s}}\right\|_{L^{2}(\omega)}$. Applying the elliptic regularity, we conclude that $\|V-\tilde{V}\|_{H^{2}(\Omega)} \leqslant$ $C\left(\left\|N_{\mathrm{s}}\right\|_{L^{2}(\omega)},\left\|\tilde{N}_{\mathrm{s}}\right\|_{L^{2}(\omega)}\right)\left\|N_{\mathrm{s}}-\tilde{N}_{\mathrm{s}}\right\|_{L^{2}(\Omega)}$ and the proof of Proposition 2.1 is complete.

Remark 2.6. We can also solve this problem by assuming that $u \in L^{2}(\omega)$ is given such that $u \geqslant 0$. More precisely, the system (1.2), (1.3) is now written

$$
\begin{aligned}
\frac{1}{2} \partial_{z}^{2} \chi_{k}+V \chi_{k} & =\epsilon_{k}, \\
-\Delta_{x, z} V & =u \sum_{k}\left|\chi_{k}\right|^{2} \mathrm{e}^{-\epsilon_{k}} .
\end{aligned}
$$

Following the same idea as that above, a weak solution of this system in the affine space $V_{0}+H^{1}(\Omega)$ is the unique minimizer with respect to $V$ of the convex functional:

$$
J(V)=\frac{1}{2} \iint_{\Omega}\left|\nabla_{x} V\right|^{2} \mathrm{~d} x \mathrm{~d} z+\int_{\omega} u \sum_{k} \mathrm{e}^{-\epsilon_{k}[V]} \mathrm{d} x
$$


(in fact, for $H^{1}$ potentials, it is not guaranteed that this functional takes finite values; to circumvent this difficulty, one can instead solve an auxiliary problem where the exponential is truncated for negative arguments, then estimate its solution and show that it is non-negative). As before, we have $V \in H^{2}(\Omega)$ for $u \in L^{2}(\omega)$.

Proof of Proposition 1.4. We consider the stationary problem (1.12), (1.13). First, we remark that the stationary drift-diffusion equation and the boundary conditions gives

$$
\begin{array}{rlrl}
-\operatorname{div}\left(\sum_{k} \mathrm{e}^{-\epsilon_{k}^{\infty}} \nabla_{x} u\right) & =0 \quad & \text { for } x \in \omega, \\
u & =u^{\infty} \quad \text { for } x \in \partial \omega,
\end{array}
$$

Thus, $u=u^{\infty}$. Then (1.12) can be written

$$
\begin{aligned}
-\frac{1}{2} \partial_{z}^{2} \chi_{k}^{\infty}+V^{\infty} \chi_{k}^{\infty} & =\epsilon_{k}^{\infty} \chi_{k}^{\infty} \\
-\Delta_{x, z} V^{\infty} & =u^{\infty} \sum_{k}\left|\chi_{k}^{\infty}\right|^{2} \mathrm{e}^{-\epsilon_{k}^{\infty}} .
\end{aligned}
$$

And the solution of this Schrödinger-Poisson system is the minimum of the convex functional (see Remark 2.6):

$$
J(V)=\frac{1}{2} \iint_{\Omega}\left|\nabla_{x, z} V\right|^{2} \mathrm{~d} x \mathrm{~d} z+\int_{\omega} u^{\infty} \sum_{k} \mathrm{e}^{-\epsilon_{k}[V]} \mathrm{d} x,
$$

where $\left(\epsilon_{k}[V]\right)_{p \geqslant 1}$ are the eigenvalues of the Hamiltonian, i.e. satisfy (1.2).

\subsection{Proof of Theorem 1.2}

The proof of existence and uniqueness relies on a contraction argument in the spirit of [25]. First, we define the map $F: N_{\mathrm{s}} \mapsto \hat{N}_{\mathrm{s}}$ as follows.

Step 1. For a given $N_{\mathrm{s}} \geqslant 0$, solve the Schrödinger-Poisson system (2.1) as in $\S 2.4$. From the $V \in C\left([0, T], H^{2}(\Omega)\right.$ ) obtained (see Proposition 2.1), define $V_{\mathrm{s}}$ by (1.6). By Lemma A 6, $V_{\mathrm{s}}$ belongs to $C\left([0, T], H^{2}(\omega)\right)$.

Step 2. The surface potential $V_{\mathrm{s}}$ being known, solve the following parabolic equation for the unknown $\hat{N}_{\mathrm{s}}$ :

$$
\partial_{t} \hat{N}_{\mathrm{s}}-\operatorname{div}_{x}\left(\nabla_{x} \hat{N}_{\mathrm{s}}+\hat{N}_{\mathrm{s}} \nabla_{x} V_{\mathrm{s}}\right)=0
$$

with the boundary condition

$$
\hat{N}_{\mathrm{s}}(t, x)=N_{b}(x) \quad \text { for } x \in \partial \omega
$$

and the initial value

$$
\hat{N}_{\mathrm{s}}(0, x)=N_{\mathrm{s}}^{0}(x) \text { for } x \in \partial \omega .
$$

Standard results on parabolic equations $[\mathbf{2 4}]$ lead to the existence and uniqueness of the solution $\hat{N}_{\mathrm{s}}$ of $(2.25),(2.26)$. Of course, $\hat{N}_{\mathrm{s}} \geqslant 0$. The map $F$ is then defined after these two steps by $F\left(N_{\mathrm{s}}\right):=\hat{N}_{\mathrm{s}}$. 
Let us now show that $F$ is a contraction on the space $M_{a, T}$ defined by $M_{a, T}=\{n$ : $\left.\|n\|_{T} \leqslant a\right\}$, where the norm is

$$
\|n\|_{T}=\left[\max _{0 \leqslant t \leqslant T}\|n(t)\|_{L^{2}(\omega)}^{2}+\int_{0}^{T}\|n(t)\|_{H^{1}(\omega)}^{2} \mathrm{~d} t\right]^{1 / 2} .
$$

The two parameters $T$ and $a$ will be specified later. Let $N_{\mathrm{s}}$ and $\tilde{N}_{\mathrm{s}}$ be two elements of $M_{a, T}$. The difference $\delta F=F\left(\tilde{N}_{\mathrm{s}}\right)-F\left(N_{\mathrm{s}}\right)$ verifies

$$
\partial_{t} \delta F-\operatorname{div}_{x}\left(\nabla_{x} \delta F+\delta F \nabla_{x} V_{\mathrm{s}}+F\left(\tilde{N}_{\mathrm{s}}\right) \nabla_{x} \delta V_{\mathrm{s}}\right)=0,
$$

with the notation $\delta V_{\mathrm{s}}=V_{\mathrm{s}}-\tilde{V}_{\mathrm{s}}$. The boundary conditions become

$$
\delta F(0, x)=0, \quad \forall x \in \omega ; \quad \delta F(t, x)=0, \quad \forall x \in \partial \omega, t \in[0, T] .
$$

Multiplying (2.28) by $\delta F$ and integrating on $\omega$, after an integration by parts we obtain

$$
\frac{1}{2} \frac{\mathrm{d}}{\mathrm{d} t} \int_{\omega}|\delta F|^{2} \mathrm{~d} x+\int_{\omega}\left|\nabla_{x}(\delta F)\right|^{2} \mathrm{~d} x+\int_{\omega} \nabla_{x}(\delta F)\left(\delta F \nabla_{x} V_{\mathrm{s}}+F\left(\tilde{N}_{\mathrm{s}}\right) \nabla_{x}\left(\delta V_{\mathrm{s}}\right)\right) \mathrm{d} x=0 .
$$

The Cauchy-Schwarz inequality applied to the third term leads to

$$
\frac{1}{2} \frac{\mathrm{d}}{\mathrm{d} t}\|\delta F\|_{L^{2}}^{2}+\left\|\nabla_{x}(\delta F)\right\|_{L^{2}}^{2} \leqslant\left\|\nabla_{x}(\delta F)\right\|_{L^{2}}\left(\left\|\delta F \nabla_{x} V_{\mathrm{s}}\right\|_{L^{2}}+\left\|F\left(\tilde{N}_{\mathrm{s}}\right) \nabla_{x}\left(\delta V_{\mathrm{s}}\right)\right\|_{L^{2}}\right) .
$$

Thus,

$$
\begin{aligned}
\frac{\mathrm{d}}{\mathrm{d} t}\|\delta F\|_{L^{2}}^{2}+\left\|\nabla_{x}(\delta F)\right\|_{L^{2}}^{2} & \leqslant 2\left\|\delta F \nabla_{x} V_{\mathrm{s}}\right\|_{L^{2}}^{2}+2\left\|F\left(\tilde{N}_{\mathrm{s}}\right) \nabla_{x}\left(\delta V_{\mathrm{s}}\right)\right\|_{L^{2}}^{2} \\
& \leqslant 2\|\delta F\|_{L^{4}}^{2}\left\|\nabla_{x} V_{\mathrm{s}}\right\|_{L^{4}}^{2}+2\left\|F\left(\tilde{N}_{\mathrm{s}}\right)\right\|_{L^{4}}^{2}\left\|\nabla_{x}\left(\delta V_{\mathrm{s}}\right)\right\|_{L^{4}}^{2} .
\end{aligned}
$$

Besides, we have

$$
\left|\nabla_{x} V_{\mathrm{s}}\right|=\frac{\left.\left|\sum_{k} \int_{0}^{1}\right| \chi_{k}\right|^{2} \nabla_{x} V \mathrm{e}^{-\epsilon_{k}} \mathrm{~d} z \mid}{\sum_{k} \mathrm{e}^{-\epsilon_{k}}} \leqslant\left|S_{2}(t, x)\right| \int_{0}^{1}\left|\nabla_{x} V\right| \mathrm{d} z
$$

where $S_{2}$ is defined by (2.10). From Proposition 2.1 and the fact that $N_{\mathrm{s}} \in M_{a, T}$, we deduce that

$$
\max _{0 \leqslant t \leqslant T}\|V(t)\|_{H^{2}(\Omega)} \leqslant C_{1}(a),
$$

where $C_{1}(a)$ is a constant only depending on $a$. From Lemma A 6 and the embedding $H^{2}(\Omega) \hookrightarrow L^{\infty}(\Omega)$, we deduce the pointwise-in-time inequalities

$$
\max _{0 \leqslant t \leqslant T}\left(\left\|S_{2}(t)\right\|_{L^{\infty}}+\left\|V_{\mathrm{s}}(t)\right\|_{H^{2}(\omega)}\right) \leqslant C_{2}(a) .
$$

From Lemma A 6 and Proposition 2.1, we know that there exists a constant $C_{2}(a)$ such that

$$
\left\|\nabla_{x}\left(\delta V_{\mathrm{s}}\right)\right\|_{L^{4}} \leqslant C\left\|\delta V_{\mathrm{s}}\right\|_{H^{2}(\omega)} \leqslant C_{2}(a)\left\|\delta N_{\mathrm{s}}\right\|_{L^{2}(\omega)} .
$$


Inserting the above inequalities into (2.29), we obtain the inequality

$$
\frac{\mathrm{d}}{\mathrm{d} t}\|\delta F\|_{L^{2}}^{2}+\left\|\nabla_{x}(\delta F)\right\|_{L^{2}}^{2} \leqslant C_{3}(a)\left(\|\delta F\|_{L^{4}}^{2}+\left\|F\left(\tilde{N}_{\mathrm{s}}\right)\right\|_{L^{4}}^{2}\left\|\delta N_{\mathrm{s}}\right\|_{L^{2}}^{2}\right) .
$$

The Gagliardo-Nirenberg inequality leads to

$$
\frac{\mathrm{d}}{\mathrm{d} t}\|\delta F\|_{L^{2}}^{2}+\frac{1}{2}\left\|\nabla_{x}(\delta F)\right\|_{L^{2}}^{2} \leqslant C_{4}(a)\left(\|\delta F\|_{L^{2}}^{2}+\left\|F\left(\tilde{N}_{\mathrm{s}}\right)\right\|_{L^{4}}^{2}\left\|\delta N_{\mathrm{s}}\right\|_{L^{2}}^{2}\right) .
$$

Taking $\tilde{N}_{\mathrm{s}}=0$ in the above inequality leads to

$$
\begin{aligned}
& \frac{\mathrm{d}}{\mathrm{d} t}\left\|F\left(N_{\mathrm{s}}\right)-F(0)\right\|_{L^{2}}^{2}+\frac{1}{2}\left\|\nabla_{x}\left(F\left(N_{\mathrm{s}}\right)-F(0)\right)\right\|_{L^{2}}^{2} \\
& \quad \leqslant C_{4}(a)\left(\left\|F\left(N_{\mathrm{s}}\right)-F(0)\right\|_{L^{2}}^{2}+\|F(0)\|_{L^{4}}^{2}\left\|N_{\mathrm{s}}\right\|_{L^{2}}^{2}\right),
\end{aligned}
$$

which implies that

$$
\begin{aligned}
\left\|F\left(N_{\mathrm{s}}\right)(t)-F(0)(t)\right\|_{L^{2}}^{2} \leqslant \| F\left(N_{\mathrm{s}}\right)(0) & -F(0)(0) \|_{L^{2}}^{2} \mathrm{e}^{C_{4}(a) t} \\
& +C_{4}(a)\|F(0)\|_{L^{4}}^{2} \int_{0}^{t}\left\|N_{\mathrm{s}}(\tau)\right\|_{L^{2}}^{2} \mathrm{e}^{C_{4}(a)(t-\tau)} \mathrm{d} \tau .
\end{aligned}
$$

We then obtain

$$
\left\|F\left(N_{\mathrm{s}}\right)\right\|_{T} \leqslant C_{5}(a) \mathrm{e}^{C_{5}(a) T}
$$

where $\|\cdot\|_{T}$ is defined in (2.27) and $C_{5}$ depends only on $a$. Of course, since $N_{\mathrm{s}}$ and $\tilde{N}_{\mathrm{s}}$ play the same role, we obviously have

$$
\left\|F\left(\tilde{N}_{\mathrm{s}}\right)\right\|_{T} \leqslant C_{5}(a) \mathrm{e}^{C_{5}(a) T} .
$$

Let us now go back to (2.30), which, after a Gronwall inequality, yields

$$
\begin{aligned}
\|\delta F(t)\|_{L^{2}}^{2} & \leqslant C_{4}(a)\left\|\delta N_{\mathrm{s}}\right\|_{T}^{2} \int_{0}^{t} \mathrm{e}^{C_{4}(a)(t-\tau)}\left\|F\left(\tilde{N}_{\mathrm{s}}\right)(\tau)\right\|_{L^{4}}^{2} \mathrm{~d} \tau \\
& \leqslant C_{4}(a) \mathrm{e}^{C_{4}(a) t}\left\|\delta N_{\mathrm{s}}\right\|_{T}^{2} \int_{0}^{t}\left\|F\left(\tilde{N}_{\mathrm{s}}\right)(\tau)\right\|_{L^{2}}\left\|\nabla_{x} F\left(\tilde{N}_{\mathrm{s}}\right)(\tau)\right\|_{L^{2}} \mathrm{~d} \tau \\
& \leqslant C_{4}(a) \mathrm{e}^{C_{4}(a) t}\left\|\delta N_{\mathrm{s}}\right\|_{T}^{2} \sqrt{T}\left\|F\left(\tilde{N}_{\mathrm{s}}\right)\right\|_{T}^{2} .
\end{aligned}
$$

We then deduce from (2.31) that

$$
\|\delta F(t)\|_{T} \leqslant C_{6}(a) T^{1 / 4} \mathrm{e}^{C_{6}(a) T}\left\|\delta N_{\mathrm{s}}\right\|_{T} .
$$

Let us now take $a=2\|F(0)\|_{1}$ and choose the parameter $T \leqslant 1$ small enough that $C_{6}(a) T^{1 / 4} \mathrm{e}^{C_{6}(a) T} \leqslant \frac{1}{2}$. Since $\|\cdot\|_{T}$ is increasing with respect to $T$, it is readily seen that $F$ leaves $M_{a, T}$ invariant and is a contraction on this set. We have then constructed a unique solution on a time-interval $T_{0}$ which depends only on the $L^{2}$ norm of the initial datum and on the $H^{1 / 2}(\partial \omega)$ norm of the boundary values for $N_{\mathrm{s}}$ and $V_{\mathrm{s}}$. In order to construct a global solution, we take $T_{0}$ as the origin and prove as above the existence and uniqueness of the solution on $\left[T_{0}, 2 T_{0}\right]$. This is made possible due to the locally uniformin-time $L^{2}$ a priori estimate on the self-consistent solution, given in Proposition 2.3. Then we construct the solution $\left[2 T_{0}, 3 T_{0}\right]$ until covering completely the interval $[0, T]$. 


\section{Long-time behaviour}

The study of the exponential convergence to the equilibrium is established in two steps. First we prove the convergence towards 0 as $t \rightarrow+\infty$ and the decrease in the relative entropy defined by

$$
\begin{array}{r}
W(t)=\sum_{k} \int_{\omega}\left(\rho_{k} \log \left(\rho_{k} / \rho_{k}^{\infty}\right)-\rho_{k}+\rho_{k}^{\infty}\right) \mathrm{d} x+\frac{1}{2} \iint_{\Omega}\left|\nabla_{x, z}\left(V-V^{\infty}\right)\right|^{2} \mathrm{~d} x \mathrm{~d} z \\
+\int_{\omega} \sum_{k} u \mathrm{e}^{-\epsilon_{k}}\left(\epsilon_{k}[V]-\epsilon_{k}\left[V^{\infty}\right]-\int_{0}^{1}\left|\chi_{k}\right|^{2}\left(V-V^{\infty}\right) \mathrm{d} z\right) \mathrm{d} x
\end{array}
$$

where we define $\rho_{k}^{\infty}=u^{\infty} \mathrm{e}^{-\epsilon_{k}^{\infty}}$. We define

$$
n=N-N^{\infty}, \quad v=V-V^{\infty}, \quad v_{\mathrm{s}}=V_{\mathrm{s}}-V_{\mathrm{s}}^{\infty}, \quad n_{\mathrm{s}}=N_{\mathrm{s}}-N_{\mathrm{s}}^{\infty} .
$$

We deduce that

$$
\left.\begin{array}{rl}
\partial_{t} n_{\mathrm{s}}-\operatorname{div}_{x}\left(\nabla_{x} n_{\mathrm{s}}+N_{\mathrm{s}}^{\infty} \nabla_{x} v_{\mathrm{s}}+n_{\mathrm{s}} \nabla_{x} V_{\mathrm{s}}^{\infty}+n_{\mathrm{s}} \nabla_{x} v_{\mathrm{s}}\right)=0, \\
-\Delta_{x, z} v=n .
\end{array}\right\}
$$

Next we consider a quadratic approximation of the relative entropy and prove its exponential convergence to 0 as $t \rightarrow+\infty$.

In the following, $C$ denotes a positive constant depending only on the data, and $\varepsilon$ denotes an arbitrarily small positive constant.

\subsection{Convergence of the relative entropy}

This section is devoted to the following preliminary result.

Proposition 3.1. Under Assumptions 1.1 and 1.3, the solution of the drift-diffusionSchrödinger-Poisson system (1.1)-(1.11) is such that

(i) the relative entropy $W$ defined by (3.1) is decreasing and

$$
\lim _{t \rightarrow+\infty} W(t)=0
$$

(ii) we have $n_{\mathrm{s}} \rightarrow 0$ in $L^{1}(\omega)$ and $v \rightarrow 0$ in $H^{1}(\omega)$ as $t \rightarrow+\infty$.

Proof. This proof is based on an idea developed in [18]. Let $\left(N_{\mathrm{s}}^{\infty}, V^{\infty}\right)$ solve the stationary problem (1.12). We deduce from (2.7) that the relative entropy satisfies

$$
\frac{\mathrm{d}}{\mathrm{d} t} W(t)=-D(t)
$$

where $D$ is given by (2.8). Then, for all $t \geqslant 0$, we have

$$
W(t)+\int_{0}^{t} D(\tau) \mathrm{d} \tau=W(0)
$$


which implies that there exists a sequence $t_{j} \rightarrow+\infty$ such that

$$
D\left(t_{j}\right) \rightarrow 0 \text { as } j \rightarrow+\infty .
$$

Now, straightforward calculations using $N_{\mathrm{s}}=u \mathrm{e}^{-V_{\mathrm{s}}}$ give

$$
D=\int_{\omega}\left(4\left|\nabla_{x} \sqrt{N_{\mathrm{s}}}\right|^{2}+2 \nabla_{x} N_{\mathrm{s}} \cdot \nabla_{x} V_{\mathrm{s}}+N_{\mathrm{s}}\left|\nabla_{x} V_{\mathrm{s}}\right|^{2}\right) \mathrm{d} x .
$$

After an integration by parts, we get

$$
\int_{\omega} \nabla_{x} N_{\mathrm{s}} \cdot \nabla_{x} V_{\mathrm{s}} \mathrm{d} x=-\int_{\omega} N_{\mathrm{s}} \Delta_{x} V_{\mathrm{s}} \mathrm{d} x+\int_{\partial \omega} N_{\mathrm{s}} \partial_{\nu} V_{\mathrm{s}} \mathrm{d} \sigma
$$

where $\nu(x)$ denotes the outward unitary normal vector at $x \in \partial \omega$ and $\mathrm{d} \sigma$ the surface measure on $\partial \omega$ induced by the Lebesgue measure. Therefore, we deduce from (3.6) that

$$
\begin{aligned}
4\left\|\nabla_{x} \sqrt{N_{\mathrm{s}}}\right\|_{L^{2}}^{2} & \leqslant D+2 \int N_{\mathrm{s}} \Delta_{x} V_{\mathrm{s}} \mathrm{d} x-2 \int_{\partial \omega} N_{\mathrm{s}} \partial_{\nu} V_{\mathrm{s}} \mathrm{d} \sigma \\
& \leqslant D+8 \int_{\omega} N_{\mathrm{s}} S_{2} \mathrm{~d} x-2 \int_{\partial \omega \times(0,1)} N \partial_{\nu} V \mathrm{~d} \sigma \mathrm{d} z \\
& \leqslant D+8\left\|N_{\mathrm{s}}\right\|_{L^{4}}\left\|S_{2}\right\|_{L^{4 / 3}}+2\left\|N_{b}\right\|_{L^{\infty}}\|V\|_{H^{2}} \\
& \leqslant D+C\left\|N_{\mathrm{s}}\right\|_{L^{4}}+C\|N\|_{L^{2}}+C,
\end{aligned}
$$

where we recall that $S_{2}$ is given by (2.10) and satisfies (2.11). Besides, it is readily seen that $N \leqslant N_{\mathrm{s}} S_{1}$, where $S_{1}$ is given in (2.10) and satisfies (2.11). Therefore, $\|N\|_{L^{2}} \leqslant$ $C\left\|N_{\mathrm{s}}\right\|_{L^{4}}$. We conclude from the above inequality that

$$
4\left\|\nabla_{x} \sqrt{N_{\mathrm{s}}}\right\|_{L^{2}}^{2} \leqslant D+C\left\|N_{\mathrm{s}}\right\|_{L^{4}}+C .
$$

Applying a Gagliardo-Nirenberg inequality to the function $\sqrt{N_{\mathrm{s}}}$ on the right-hand side, we obtain (for any $\varepsilon>0$ )

$$
4\left\|\nabla_{x} \sqrt{N_{\mathrm{s}}}\right\|_{L^{2}}^{2} \leqslant D+C\left\|N_{\mathrm{s}}\right\|_{L^{1}}^{1 / 2}\left\|\sqrt{N_{\mathrm{s}}}\right\|_{H^{1}}+C \leqslant D+C_{\varepsilon}\left\|N_{\mathrm{s}}\right\|_{L^{1}}+\varepsilon\left\|\nabla_{x} \sqrt{N_{\mathrm{s}}}\right\|_{L^{2}}^{2}+C,
$$

which leads, in view of (2.9), to the inequality

$$
\left\|\nabla_{x} \sqrt{N_{\mathrm{s}}}\right\|_{L^{2}}^{2}(t) \leqslant C(D(t)+1) .
$$

By evaluating (3.5) and (3.7) at $t=t_{j}$, we deduce the boundedness in $H^{1}(\omega)$ of the sequence $\left(\sqrt{N_{\mathrm{s}}\left(t_{j}\right)}\right)_{j}$. Because of the compactness embedding of $H^{1}(\omega)$ into $L^{4}(\omega)$, we can assume without loss of generality that there exists $\bar{N}_{\mathrm{s}}$ belonging to $L^{2}(\omega)$ such that $\sqrt{\bar{N}_{\mathrm{s}}} \in H^{1}(\omega)$ and

$$
N_{\mathrm{s}}\left(t_{j}\right) \rightarrow \bar{N}_{\mathrm{s}} \quad \text { in } L^{2}(\omega)
$$


Thanks to the properties of the trace of $H^{1}(\omega)$ functions and the compact embedding $H^{1 / 2}(\partial \omega) \hookrightarrow L^{4}(\partial \omega)$, we have $\left.\bar{N}_{\mathrm{s}}\right|_{\partial \omega}=N_{b}$. From Proposition 2.1, we know that the mapping $N_{\mathrm{s}} \mapsto V$ defined by

$$
\begin{gathered}
-\frac{1}{2} \partial_{z}^{2} \chi_{k}+V \chi_{k}=\epsilon_{k} \chi_{k}, \quad k \geqslant 1, \\
-\Delta_{x, z} V=N=N_{\mathrm{s}} \sum_{k} \frac{\left|\chi_{k}\right|^{2} \mathrm{e}^{-\epsilon_{k}}}{\mathcal{Z}}
\end{gathered}
$$

(with the boundary conditions of $V$ in (1.11)) is well posed for $N_{\mathrm{s}} \in L^{2}(\omega)$ such that $N_{\mathrm{s}} \geqslant 0$ a.e. and is continuous from $L^{2}(\omega)$ into $H^{2}(\Omega)$. Moreover, by Lemma A 6 we also know that the mapping $V \mapsto V_{\mathrm{s}}$ defined by

$$
\begin{aligned}
& V_{\mathrm{s}}=-\log \left(\sum_{k} \mathrm{e}^{-\epsilon_{k}}\right), \\
& -\frac{1}{2} \partial_{z}^{2} \chi_{k}+V \chi_{k}=\epsilon_{k} \chi_{k}
\end{aligned}
$$

is continuous from $H^{2}(\Omega)$ to $H^{2}(\omega)$. It follows that

$$
\exists \bar{V}_{\mathrm{s}} \in H^{2}(\omega) \text { such that } V_{\mathrm{s}}\left(t_{j}\right) \rightarrow \bar{V}_{\mathrm{s}} \text { in } H^{2}(\omega) \subset C(\bar{\omega}) .
$$

Hence,

$$
u\left(t_{j}\right)=N_{\mathrm{s}}\left(t_{j}\right) \mathrm{e}^{V_{\mathrm{s}}\left(t_{j}\right)} \rightarrow \bar{N}_{\mathrm{s}} \mathrm{e}^{\bar{V}_{\mathrm{s}}} \quad \text { in } L^{2}(\omega)
$$

Now (3.5) and (3.8) imply that, for any $h \in\left(L^{4}(\omega)\right)^{2}$, we have

$$
\begin{aligned}
\left|\int_{\omega} \nabla_{x}\left(N_{\mathrm{s}}\left(t_{j}\right) \mathrm{e}^{V_{\mathrm{s}}\left(t_{j}\right)}\right) h \mathrm{~d} x\right|= & \left|\int_{\omega} \nabla_{x} u\left(t_{j}\right) h \mathrm{~d} x\right| \\
\leqslant & \left(\int_{\omega} \mathrm{e}^{-V_{\mathrm{s}}\left(t_{j}\right)} \frac{\left|\nabla_{x} u\left(t_{j}\right)\right|^{2}}{u\left(t_{j}\right)} \mathrm{d} x\right) \\
& \quad \times\left\|N_{\mathrm{s}}\left(t_{j}\right) \mathrm{e}^{2 V_{\mathrm{s}}\left(t_{j}\right)}\right\|_{L^{2}(\omega)}^{1 / 2}\|h\|_{L^{4}(\omega)} \rightarrow 0 \quad \text { as } j \rightarrow+\infty .
\end{aligned}
$$

Taking into account (3.9), we deduce that $\bar{N}_{\mathrm{s}} \mathrm{e}^{\bar{V}_{\mathrm{s}}}$ is constant in $\omega$. Since $\left.\bar{N}_{\mathrm{s}}\right|_{\partial \omega}=N_{b}$ and $\left.\bar{V}_{\mathrm{s}}\right|_{\partial \omega}=V_{\mathrm{s}}^{\infty}$, Assumption 1.3 implies $\bar{N}_{\mathrm{s}} \mathrm{e}^{\bar{V}_{\mathrm{s}}}=u^{\infty}$. Thus, $\left(\bar{N}_{\mathrm{s}}, \bar{V}_{\mathrm{s}}\right)$ can be identified as the unique solution of the stationary Schrödinger-Poisson system (see Remark 2.6):

$$
\bar{N}_{\mathrm{s}}=N_{\mathrm{s}}^{\infty}, \quad \bar{V}_{\mathrm{s}}=V_{\mathrm{s}}^{\infty} \quad \text { and analogously } \quad V\left(t_{j}\right) \rightarrow V^{\infty} \quad \text { as } j \rightarrow+\infty .
$$

Since the function $W$ is decreasing, we have

$$
\lim _{t \rightarrow+\infty} W(t)=\lim _{j \rightarrow+\infty} W\left(t_{j}\right)=0 .
$$

Consequently, $\|v(t)\|_{H^{1}(\Omega)} \rightarrow 0$ and $\left\|n_{\mathrm{s}}\right\|_{L^{1}(\omega)} \rightarrow 0$ as $t \rightarrow+\infty$ by a Poincaré inequality and the following Csiszár-Kullback inequality $[\mathbf{3}, \mathbf{1 1}, \mathbf{2 3}]$ : for all $n_{1}, n_{2} \in L^{1}(\omega), n_{1} \geqslant$ 0 a.e., $n_{2} \geqslant 0$ a.e. with

$$
\int_{\omega} n_{1} \mathrm{~d} x=\int_{\omega} n_{2} \mathrm{~d} x=N_{0}
$$

we have

$$
\left\|n_{1}-n_{2}\right\|_{L^{1}(\omega)}^{2} \leqslant 2 N_{0} \int_{\omega} n_{1} \log \frac{n_{1}}{n_{2}} \mathrm{~d} x .
$$




\subsection{Exponential convergence}

This section is devoted to the proof of the main result of this paper, i.e. the exponential convergence of the surface density $N_{\mathrm{s}}$ and the electrostatic potential $V$ to the equilibrium functions. We will consider the differences $n, n_{\mathrm{s}}, v$ and $v_{\mathrm{s}}$ defined in (3.2) and introduce the quadratic approximation of the relative entropy:

$L(t)=\frac{1}{2} \int_{\omega} \frac{\left(n_{\mathrm{s}}\right)^{2}}{N_{\mathrm{s}}^{\infty}} \mathrm{d} x+\int_{\omega} n_{\mathrm{s}} v_{\mathrm{s}} \mathrm{d} x-\frac{1}{2} \iint_{\Omega}|\nabla v|^{2} \mathrm{~d} x \mathrm{~d} z+\int_{\omega} N_{\mathrm{s}}^{\infty} v_{\mathrm{s}} \mathrm{d} x-\iint_{\Omega} N^{\infty} v \mathrm{~d} x \mathrm{~d} z$.

Since the Poisson equation gives

$$
\iint_{\Omega} n v \mathrm{~d} x \mathrm{~d} z=\iint_{\Omega}|\nabla v|^{2} \mathrm{~d} x \mathrm{~d} z
$$

we can rewrite the above equation as

$$
L(t)=\frac{1}{2} \int_{\omega} \frac{\left(n_{\mathrm{s}}\right)^{2}}{N_{\mathrm{s}}^{\infty}} \mathrm{d} x+\frac{1}{2} \iint_{\Omega}|\nabla v|^{2} \mathrm{~d} x \mathrm{~d} z+\int_{\omega} N_{\mathrm{s}} v_{\mathrm{s}} \mathrm{d} x-\iint_{\Omega} N v \mathrm{~d} x \mathrm{~d} z .
$$

In order to prove Theorem 1.5, we need the following three technical lemmas, which we go on to prove in $\S 3.2 .2$.

Lemma 3.2. Consider a weak solution of (1.2)-(1.11). Then, for all $t \geqslant 0$, we have

$$
\frac{1}{2} \int_{\omega} \frac{\left(n_{\mathrm{s}}\right)^{2}}{N_{\mathrm{s}}^{\infty}} \mathrm{d} x+\frac{1}{2} \iint_{\Omega}|\nabla v|^{2} \mathrm{~d} x \mathrm{~d} z \leqslant L(t) \leqslant \frac{1}{2} \int_{\omega} \frac{\left(n_{\mathrm{s}}\right)^{2}}{N_{\mathrm{s}}^{\infty}} \mathrm{d} x+\int_{\omega} n_{\mathrm{s}} v_{\mathrm{s}} \mathrm{d} x .
$$

Lemma 3.3. Let $V$ and $\underline{V}$ belong to $L^{2}(0,1)$ and $V_{\mathrm{s}}, \underline{V_{\mathrm{s}}}$ be defined by

$$
V_{\mathrm{s}}=-\log \sum_{k} \exp \left(-\epsilon_{k}[V]\right) \quad \text { and } \quad \underline{V_{\mathrm{s}}}=-\log \sum_{k} \exp \left(-\epsilon_{k}[\underline{V}]\right) .
$$

Then, by setting $v=V-\underline{V}$ and $v_{\mathrm{s}}=V_{\mathrm{s}}-\underline{V_{\mathrm{s}}}$, we have

$$
\left|\nabla_{x} v_{\mathrm{s}}\right|^{2} \leqslant C_{1} \exp \left\{C_{2}\left(\|\underline{V}\|_{L_{z}^{2}(0,1)}+\|v\|_{L_{z}^{2}(0,1)}\right)\right\}\left(\left\langle\left|\nabla_{x} v\right|\right\rangle^{2}+\langle|v|\rangle^{2}\left\langle\left|\nabla_{x} \underline{V}\right|\right\rangle^{2}\right),
$$

where $C_{1}$ and $C_{2}$ are two positive constants.

Lemma 3.4. Consider a weak solution of (1.2)-(1.11). There then exist two nonnegative constants $C_{1}$ and $C_{2}$ such that, for all $t \geqslant 0$,

$$
\int_{\omega} \frac{\left(n_{\mathrm{s}}\right)^{2}}{N_{\mathrm{s}}^{\infty}}\left|\nabla_{x} v_{\mathrm{s}}\right|^{2} \mathrm{~d} x \leqslant \frac{1}{2} \int_{\omega} N_{\mathrm{s}}^{\infty}\left|\nabla_{x}\left(\frac{n_{\mathrm{s}}}{N_{\mathrm{s}}^{\infty}}+v_{\mathrm{s}}\right)\right|^{2} \mathrm{~d} x+C_{1} L(t)^{4}+C_{2} L(t)\|v\|_{H^{1}(\Omega)},
$$

where $L$ is defined in (3.11). Moreover, we have

$$
\left\|v_{\mathrm{s}}\right\|_{L^{6}(\Omega)} \leqslant C\|v\|_{H^{1}(\Omega)},
$$

for a non-negative constant $C$. 


\subsubsection{Proof of Theorem 1.5}

From (3.10) and the Poisson equation, we deduce that

$$
\frac{\mathrm{d}}{\mathrm{d} t} L(t)=\int_{\omega} \partial_{t} n_{\mathrm{s}}\left(\frac{n_{\mathrm{s}}}{N_{\mathrm{s}}^{\infty}}+v_{\mathrm{s}}\right) \mathrm{d} x+\int_{\omega} N_{\mathrm{s}} \partial_{t} v_{\mathrm{s}} \mathrm{d} x-\iint_{\Omega} N \partial_{t} v \mathrm{~d} x \mathrm{~d} z .
$$

Furthermore, $\mathrm{e}^{-V_{\mathrm{s}}}=\sum_{k} \mathrm{e}^{-\epsilon_{k}}=\mathcal{Z}$ and $\partial_{t} \epsilon_{k}=\left\langle\left|\chi_{k}\right|^{2} \partial_{t} v\right\rangle$ imply that

$$
\partial_{t} v_{\mathrm{s}}=\frac{1}{\mathcal{Z}} \sum_{k}\left\langle\left|\chi_{k}\right|^{2} \partial_{t} v\right\rangle \mathrm{e}^{-\epsilon_{k}}
$$

Hence,

$$
\int_{\omega} N_{\mathrm{s}} \partial_{t} v_{\mathrm{s}} \mathrm{d} x=\iint_{\Omega} N \partial_{t} v \mathrm{~d} x \mathrm{~d} z
$$

With (3.3) and after an integration by parts, we get

$$
\frac{\mathrm{d}}{\mathrm{d} t} L(t)=-\int_{\omega}\left(\nabla_{x} n_{\mathrm{s}}+N_{\mathrm{s}}^{\infty} \nabla_{x} v_{\mathrm{s}}+n_{\mathrm{s}} \nabla_{x} V_{\mathrm{s}}^{\infty}+n_{\mathrm{s}} \nabla_{x} v_{\mathrm{s}}\right) \cdot \nabla_{x}\left(\frac{n_{\mathrm{s}}}{N_{\mathrm{s}}^{\infty}}+v_{\mathrm{s}}\right) \mathrm{d} x .
$$

Since $\nabla_{x} N_{\mathrm{s}}^{\infty}+N_{\mathrm{s}}^{\infty} \nabla_{x} V_{\mathrm{s}}^{\infty}=0$, we deduce that

$$
\frac{\mathrm{d}}{\mathrm{d} t} L(t)=-\int_{\omega} N_{\mathrm{s}}^{\infty}\left|\nabla_{x}\left(\frac{n_{\mathrm{s}}}{N_{\mathrm{s}}^{\infty}}+v_{\mathrm{s}}\right)\right|^{2} \mathrm{~d} x-\int_{\omega} n_{\mathrm{s}} \nabla_{x} v_{\mathrm{s}} \cdot \nabla_{x}\left(\frac{n_{\mathrm{s}}}{N_{\mathrm{s}}^{\infty}}+v_{\mathrm{s}}\right) \mathrm{d} x .
$$

Now we will show that the second term on the right-hand side of (3.14) can be controlled by the first one for long time. From Lemma 3.4, we deduce that

$$
\begin{aligned}
-\int_{\omega} n_{\mathrm{s}} \nabla_{x} v_{\mathrm{s}} \cdot \nabla_{x}( & \left.\frac{n_{\mathrm{s}}}{N_{\mathrm{s}}^{\infty}}+v_{\mathrm{s}}\right) \mathrm{d} x \\
& \leqslant \frac{1}{2} \int_{\omega} \frac{\left(n_{\mathrm{s}}\right)^{2}}{N_{\mathrm{s}}^{\infty}}\left|\nabla_{x} v_{\mathrm{s}}\right|^{2} \mathrm{~d} x+\frac{1}{2} \int_{\omega} N_{\mathrm{s}}^{\infty}\left|\nabla_{x}\left(\frac{n_{\mathrm{s}}}{N_{\mathrm{s}}^{\infty}}+v_{\mathrm{s}}\right)\right|^{2} \mathrm{~d} x \\
& \leqslant \frac{3}{4} \int_{\omega} N_{\mathrm{s}}^{\infty}\left|\nabla_{x}\left(\frac{n_{\mathrm{s}}}{N_{\mathrm{s}}^{\infty}}+v_{\mathrm{s}}\right)\right|^{2} \mathrm{~d} x+C_{1} L(t)^{4}+C_{2}\|v\|_{H^{1}(\Omega)} L(t) .
\end{aligned}
$$

Thanks to the Poincaré inequality and Lemma 3.2, we have

$$
\begin{aligned}
-\frac{1}{4} \int_{\omega} N_{\mathrm{s}}^{\infty}\left|\nabla_{x}\left(\frac{n_{\mathrm{s}}}{N_{\mathrm{s}}^{\infty}}+v_{\mathrm{s}}\right)\right|^{2} \mathrm{~d} x & \leqslant-\frac{1}{4} C \int_{\omega} N_{\mathrm{s}}^{\infty}\left(\frac{n_{\mathrm{s}}}{N_{\mathrm{s}}^{\infty}}+v_{\mathrm{s}}\right)^{2} \mathrm{~d} x \\
& \leqslant-\frac{1}{2} C \int_{\omega}\left(\frac{1}{2} \frac{\left(n_{\mathrm{s}}\right)^{2}}{N_{\mathrm{s}}^{\infty}}+n_{\mathrm{s}} v_{\mathrm{s}}\right) \mathrm{d} x \leqslant-\frac{1}{2} C L(t) .
\end{aligned}
$$

Hence, from (3.14) we have obtained

$$
\frac{\mathrm{d}}{\mathrm{d} t} L(t) \leqslant-C_{0} L(t)+C_{1} L(t)^{4}+C_{2}\|v\|_{H^{1}(\Omega)} L(t) .
$$


By Proposition 3.1 (ii), there exists $T>0$ such that, for all $t \geqslant T, C_{2}\|v\|_{H^{1}(\omega)}(t) \leqslant \frac{1}{2} C_{0}$. Thus, for all $t \geqslant T$,

$$
\frac{\mathrm{d}}{\mathrm{d} t} L(t) \leqslant-\frac{C_{0}}{2} L(t)+C_{1} L(t)^{4} .
$$

From (3.5) and (3.7), we see that there exists a sequence $t_{j} \rightarrow+\infty$ as $j \rightarrow+\infty$ such that the sequence $\left(\sqrt{N_{\mathrm{s}}\left(t_{j}\right)}\right)_{j \in \mathbb{N}}$ is bounded in $H^{1}(\omega)$. Up to a renumbering, we can suppose that, for all $j \in \mathbb{N}, t_{j} \geqslant T$. Moreover, by interpolation, we have

$$
\left\|\frac{n_{\mathrm{s}}}{\sqrt{N_{\mathrm{s}}^{\infty}}}\right\|_{L^{2}(\omega)} \leqslant C\left\|n_{\mathrm{s}}\right\|_{L^{1}(\omega)}^{1 / 4}\left\|n_{\mathrm{s}}\right\|_{L^{3}(\omega)}^{3 / 4} .
$$

By the Sobolev embedding of $H^{1}(\omega)$ into $L^{6}(\omega)$, we deduce that $\left\|n_{\mathrm{s}}\right\|_{L^{3}(\omega)}\left(t_{j}\right)$ is bounded. Since we have proved in Proposition 3.1 (ii) that $n_{\mathrm{s}} \rightarrow 0$ in $L^{1}(\omega)$ as $t \rightarrow+\infty$, this ensures the convergence towards 0 of $\left\|n_{\mathrm{s}} / \sqrt{N_{\mathrm{s}}^{\infty}}\right\|_{L^{2}(\omega)}\left(t_{j}\right)$ as $j \rightarrow+\infty$. Moreover, with the bound of $L$ in Lemma 3.2 we deduce that

$$
L(t) \leqslant \frac{1}{2}\left\|\frac{n_{\mathrm{s}}}{\sqrt{N_{\mathrm{s}}^{\infty}}}\right\|_{L^{2}(\omega)}^{2}+C\left\|n_{\mathrm{s}}\right\|_{L^{2}(\omega)}\left\|v_{\mathrm{s}}\right\|_{L^{6}(\omega)} .
$$

And (3.13) provides a bound of $\left\|v_{\mathrm{s}}\right\|_{L^{6}(\omega)}$ by $\|v\|_{H^{1}(\Omega)}$, which converges towards 0 as $t \rightarrow+\infty$ due to Proposition 3.1. We can now conclude that $\lim _{j \rightarrow+\infty} L\left(t_{j}\right)=0$. Hence,

$$
\exists t_{*}>0 \text { such that } C_{1} L\left(t_{*}\right)^{3} \leqslant \frac{1}{4} C_{0} .
$$

Now we define the set

$$
\mathcal{A}:=\left\{t \in\left[t_{*},+\infty\right) \text { such that } \forall s \in\left[t_{*}, t\right], C_{1} L(s)^{3} \leqslant \frac{1}{4} C_{0}\right\} .
$$

By the continuity of $L, \mathcal{A}$ is a closed set that contains $t_{*}$ from (3.17). Moreover, if $t_{0} \in \mathcal{A}$, from (3.16) we deduce that $L$ is decreasing near $t_{0}$. By continuity of $L$, it yields that $\mathcal{A}$ is open. Thus, $\mathcal{A}=\left[t_{*},+\infty\right)$, i.e.

$$
\forall t \in\left[t_{*},+\infty\right), \quad \frac{\mathrm{d}}{\mathrm{d} t} L(t) \leqslant-\frac{C_{0}}{4} L(t) .
$$

We obtain the announced result by integrating this last inequality.

\subsubsection{Proofs of the technical lemmas}

Proof of Lemma 3.2. The concavity of the function $x \mapsto \log x$ leads to the inequality

$$
v_{\mathrm{s}}=\log \left(\frac{\sum_{k} \mathrm{e}^{-\epsilon_{k}^{\infty}}}{\sum_{k} \mathrm{e}^{-\epsilon_{k}}}\right)=\log \left(\sum_{k} \frac{\mathrm{e}^{-\epsilon_{k}}}{\sum_{\ell} \mathrm{e}^{-\epsilon_{\ell}}} \mathrm{e}^{\epsilon_{k}-\epsilon_{k}^{\infty}}\right) \geqslant \sum_{k} \frac{\mathrm{e}^{-\epsilon_{k}}}{\sum_{\ell} \mathrm{e}^{-\epsilon_{\ell}}}\left(\epsilon_{k}-\epsilon_{k}^{\infty}\right) .
$$

Therefore,

$$
\begin{aligned}
N_{\mathrm{s}} v_{\mathrm{s}}-\langle N v\rangle & =N_{\mathrm{s}}\left(v_{\mathrm{s}}-\sum_{k} \frac{\mathrm{e}^{-\epsilon_{k}}}{\sum_{\ell} \mathrm{e}^{-\epsilon_{\ell}}}\left\langle\left|\chi_{k}\right|^{2} v\right\rangle\right) \\
& \geqslant \frac{N_{\mathrm{s}}}{\sum_{\ell} \mathrm{e}^{-\epsilon_{\ell}}} \sum_{k} \mathrm{e}^{-\epsilon_{k}}\left(\epsilon_{k}-\epsilon_{k}^{\infty}-\left\langle\left|\chi_{k}[V]\right|^{2} v\right\rangle\right) .
\end{aligned}
$$


The right-hand side of this inequality is exactly the third term of (2.6), which is positive. Therefore,

$$
\int_{\omega} N_{\mathrm{s}} v_{\mathrm{s}} \mathrm{d} x-\iint_{\Omega} N v \mathrm{~d} x \mathrm{~d} z \geqslant 0
$$

By exchanging the roles of $\left(N, N^{\infty}\right)$ and $\left(V, V^{\infty}\right)$, we obtain

$$
\iint_{\Omega} N^{\infty} v \mathrm{~d} x \mathrm{~d} z-\int_{\omega} N_{\mathrm{s}}^{\infty} v_{\mathrm{s}} \mathrm{d} x \geqslant 0
$$

which leads, by (3.11) and for all $t \geqslant 0$, to

$$
0 \leqslant \frac{1}{2} \int_{\omega} \frac{\left(n_{\mathrm{s}}\right)^{2}}{N_{\mathrm{S}}^{\infty}} \mathrm{d} x+\frac{1}{2} \iint_{\Omega}|\nabla v|^{2} \mathrm{~d} x \mathrm{~d} z \leqslant L(t) .
$$

This ends the proof of Lemma 3.2. Note that the sum of (3.19) and (3.20) leads to the inequality

$$
\int_{\omega} n_{\mathrm{s}} v_{\mathrm{s}} \mathrm{d} x \geqslant \iint_{\Omega} n v \mathrm{~d} x \mathrm{~d} z=\iint_{\Omega}|\nabla v|^{2} \geqslant 0
$$

Proof of Lemma 3.3. We have

$$
\nabla_{x} v_{\mathrm{s}}=\sum_{k} \frac{\mathrm{e}^{-\epsilon_{k}}}{\mathcal{Z}}\left(\partial_{x} \epsilon_{k}-\partial_{x} \underline{\epsilon_{k}}\right)+\sum_{k}\left(\frac{\mathrm{e}^{-\epsilon_{k}}}{\mathcal{Z}}-\frac{\mathrm{e}^{-\epsilon_{k}}}{\underline{\mathcal{Z}}}\right) \partial_{x} \underline{\epsilon_{k}},
$$

with the notation $\underline{\mathcal{Z}}=\sum_{\ell} \mathrm{e}^{-\underline{\epsilon}_{\ell}}$. Thus, by a Jensen inequality,

$$
\left|\nabla_{x} v_{\mathrm{s}}\right|^{2} \leqslant 2 \sum_{k} \frac{\mathrm{e}^{-\epsilon_{k}}}{\mathcal{Z}}\left|\partial_{x} \epsilon_{k}-\partial_{x} \underline{\epsilon_{k}}\right|^{2}+2\left|\sum_{k}\left(\frac{\mathrm{e}^{-\epsilon_{k}}}{\mathcal{Z}}-\frac{\mathrm{e}^{-\underline{\epsilon_{k}}}}{\underline{\mathcal{Z}}}\right) \partial_{x} \underline{\epsilon_{k}}\right|^{2} .
$$

For the first term on the right-hand side, we use the results stated in Lemmas A 2 and A 4:

$$
\begin{aligned}
\sum_{k} \frac{\mathrm{e}^{-\epsilon_{k}}}{\mathcal{Z}}\left|\partial_{x} \epsilon_{k}-\partial_{x} \underline{\epsilon_{k}}\right|^{2} & \leqslant 2 \sum_{k} \frac{\mathrm{e}^{-\epsilon_{k}}}{\mathcal{Z}}\left\langle\left|\chi_{k}\right|^{2} \nabla_{x} v\right\rangle^{2}+2 \sum_{k} \frac{\mathrm{e}^{-\epsilon_{k}}}{\mathcal{Z}}\left\langle\left(\left|\chi_{k}\right|^{2}-\left|\underline{\chi_{k}}\right|^{2}\right) \nabla_{x} \underline{V}\right\rangle^{2} \\
\leqslant & C_{1} \exp \left\{C_{2}\|V(x, \cdot)\|_{L^{2}(0,1)}\right\}\left\langle\left|\nabla_{x} v\right|\right\rangle^{2} \\
& +\int_{0}^{1} C_{1} \exp \left\{C_{2}\|\underline{V}(x, \cdot)+s v(x, \cdot)\|_{L^{2}(0,1)}\right\}\langle|v|\rangle^{2}\left\langle\left|\nabla_{x} \underline{V}\right|\right\rangle^{2} \mathrm{~d} s .
\end{aligned}
$$

Consequently, we have

$$
\sum_{k} \frac{\mathrm{e}^{-\epsilon_{k}}}{\mathcal{Z}}\left|\partial_{x} \epsilon_{k}-\partial_{x} \underline{\epsilon_{k}}\right|^{2} \leqslant C_{1} \exp \left\{C_{2}\left(\|\underline{V}\|_{L^{2}(0,1)}+\|v\|_{L^{2}(0,1)}\right)\right\}\left(\left\langle\left|\nabla_{x} v\right|\right\rangle^{2}+\langle|v|\rangle^{2}\left\langle\left|\nabla_{x} \underline{V}\right|\right\rangle^{2}\right) .
$$


We can write the second term on the right-hand side of (3.22) as

$$
\begin{aligned}
& \sum_{k}\left(\frac{\mathrm{e}^{-\epsilon_{k}}}{\mathcal{Z}}-\frac{\mathrm{e}^{\frac{\epsilon_{k}}{\mathcal{Z}}}}{\underline{\mathcal{Z}}}\right) \partial_{x} \underline{\epsilon_{k}}=\int_{0}^{1} \frac{\sum_{k}\left\langle\left|\chi_{k}^{s}\right|^{2} v\right\rangle \mathrm{e}^{-\epsilon_{k}^{s}}}{\sum_{\ell} \mathrm{e}^{-\epsilon_{\ell}^{s}}} \frac{\sum_{k}\left\langle\left|\underline{\chi_{k}}\right|^{2} \nabla_{x} \underline{V}\right\rangle \mathrm{e}^{-\epsilon_{k}^{s}}}{\sum_{\ell} \mathrm{e}^{-\epsilon_{\ell}^{s}}} \mathrm{~d} s \\
& -\int_{0}^{1} \sum_{k} \frac{\left\langle\left|\chi_{k}^{s}\right|^{2} v\right\rangle \mathrm{e}^{-\epsilon_{k}^{s}}}{\sum_{\ell} \mathrm{e}^{-\epsilon_{\ell}^{s}}}\left\langle\left|\underline{\chi_{k}}\right|^{2} \nabla_{x} \underline{V}\right\rangle \mathrm{d} s,
\end{aligned}
$$

where we use the notation $\epsilon_{k}^{s}=\epsilon_{k}[\underline{V}+s v]$ and $\chi_{k}^{s}=\chi_{k}[\underline{V}+s v]$. Thus, by applying the $L^{\infty}$ bound in the $z$ direction for $\chi_{k}^{s}$ and $\underline{\chi_{k}}$ stated in Lemma A 2, we obtain

$$
\left|\sum_{k}\left(\frac{\mathrm{e}^{-\epsilon_{k}}}{\mathcal{Z}}-\frac{\mathrm{e}^{-\epsilon_{k}}}{\underline{\mathcal{Z}}}\right) \partial_{x} \underline{\epsilon_{k}}\right|^{2} \leqslant C_{1} \exp \left\{C_{2}\left(\|\underline{V}\|_{L^{2}(0,1)}+\|v\|_{L^{2}(0,1)}\right)\right\}\langle|v|\rangle^{2}\left\langle\left|\nabla_{x} \underline{V}\right|\right\rangle^{2} .
$$

By combining (3.23) and (3.24) in (3.22), we obtain (3.12).

Proof of Lemma 3.4. From (3.12), we deduce

$$
\begin{aligned}
& \int_{\omega} \frac{\left(n_{\mathrm{s}}\right)^{2}}{N_{\mathrm{s}}^{\infty}}\left|\nabla_{x} v_{\mathrm{s}}\right|^{2} \mathrm{~d} x \\
& \quad \leqslant C_{1} \int_{\omega} \frac{\left(n_{\mathrm{s}}\right)^{2}}{N_{\mathrm{s}}^{\infty}} \exp \left\{C_{2}\left(\left\|V^{\infty}\right\|_{L_{z}^{2}(0,1)}+\|v\|_{L_{z}^{2}(0,1)}\right)\right\}\left(\left\langle\left|\nabla_{x} v\right|\right\rangle^{2}+\langle|v|\rangle^{2}\left\langle\left|\nabla_{x} V^{\infty}\right|\right\rangle^{2}\right) \mathrm{d} x
\end{aligned}
$$

Throughout the proof, $C, C_{1}$ and $C_{2}$ stand for universal constants. Since $V$ is bounded in $H^{1}(\Omega)$ uniformly in time, the Trudinger inequality implies that

$$
\exp \left(C_{2}\left(\left\|V^{\infty}\right\|_{L_{z}^{2}(0,1)}+\|v\|_{L_{z}^{2}(0,1)}\right)\right) \in L^{p}(\omega), \quad \forall p \in[1, \infty) .
$$

Thus, a Hölder inequality gives

$$
\begin{aligned}
\int_{\omega} \frac{\left(n_{\mathrm{s}}\right)^{2}}{N_{\mathrm{s}}^{\infty}} \exp \left\{C_{2}\left(\left\|V^{\infty}\right\|_{L_{z}^{2}(0,1)}+\|v\|_{L_{z}^{2}(0,1)}\right)\right\} & \left\langle\left|\nabla_{x} v\right|\right\rangle^{2} \mathrm{~d} x \\
& \leqslant C\left\|\frac{n_{\mathrm{s}}}{\sqrt{N_{\mathrm{s}}^{\infty}}}\right\|_{L^{3}(\omega)}^{2}\left\|\left\langle\left|\nabla_{x} v\right|\right\rangle\right\|_{L^{8}(\omega)}^{2} .
\end{aligned}
$$

Using the expression given in (1.7) for $n=N-N^{\infty}$, we deduce that

$$
n=n_{\mathrm{s}} \sum_{k} \frac{\left|\chi_{k}\right|^{2} \mathrm{e}^{-\epsilon_{k}}}{\mathcal{Z}}+N_{\mathrm{s}}^{\infty} \sum_{k}\left[\left(\left|\chi_{k}\right|^{2}-\left|\chi_{k}^{\infty}\right|^{2}\right) \frac{\mathrm{e}^{-\epsilon_{k}}}{\mathcal{Z}}+\left|\chi_{k}^{\infty}\right|^{2}\left(\frac{\mathrm{e}^{-\epsilon_{k}}}{\mathcal{Z}}-\frac{\mathrm{e}^{-\epsilon_{k}^{\infty}}}{\mathcal{Z}^{\infty}}\right)\right] .
$$

As we saw earlier, by defining $\epsilon_{k}^{s}=\epsilon_{k}[V+s v]$, and $\chi_{k}^{s}=\chi_{k}[V+s v]$, using Lemma A 3 we can rewrite the third term as

$$
\begin{aligned}
& \sum_{k}\left|\chi_{k}^{\infty}\right|^{2}\left(\frac{\mathrm{e}^{-\epsilon_{k}}}{\mathcal{Z}}-\frac{\mathrm{e}^{-\epsilon_{k}^{\infty}}}{\mathcal{Z}}\right) \\
&=\int_{0}^{1} \frac{\sum_{k}\left\langle\left|\chi_{k}^{s}\right|^{2} v\right\rangle \mathrm{e}^{-\epsilon_{k}^{s}}}{\sum_{\ell} \mathrm{e}^{-\epsilon_{\ell}^{s}}} \frac{\sum_{k}\left|\chi_{k}^{\infty}\right|^{2} \mathrm{e}^{-\epsilon_{k}^{s}}}{\sum_{\ell} \mathrm{e}^{-\epsilon_{\ell}^{s}}} \mathrm{~d} s-\int_{0}^{1} \sum_{k} \frac{\left\langle\left|\chi_{k}^{s}\right|^{2} v\right\rangle \mathrm{e}^{-\epsilon_{k}^{s}}}{\sum_{\ell} \mathrm{e}^{-\epsilon_{\ell}^{s}}}\left|\chi_{k}^{\infty}\right|^{2} \mathrm{~d} s
\end{aligned}
$$


Since Lemma A 2 provides a bound of the eigenvectors of the Hamiltonian $\chi_{k}$ uniformly in $k$, we deduce, by Lemmas A 2 and $\mathrm{A} 4$, that

$$
|n|(x, z) \leqslant C_{1} \exp \left\{C_{2}\left(\left\|V^{\infty}\right\|_{L_{z}^{2}(0,1)}+\|v\|_{L_{z}^{2}(0,1)}\right)\right\}\left(\left|n_{\mathrm{s}}\right|(x)+N_{\mathrm{s}}^{\infty}\|v\|_{L_{z}^{1}(0,1)}(x)\right) .
$$

Therefore, using interpolation inequalities, (3.26) and $N_{\mathrm{s}}^{\infty} \in L^{\infty}(\omega)$, one may deduce from elliptic regularity for the Poisson equation (1.3) that

$$
\|v\|_{H^{2}(\Omega)} \leqslant C\|n\|_{L^{2}(\Omega)} \leqslant C\left(\left\|n_{\mathrm{s}}\right\|_{L^{18 / 7}(\omega)}+\|v\|_{H^{1}(\Omega)}\right) .
$$

With a Gagliardo-Nirenberg inequality and (3.28), we obtain

$$
\begin{aligned}
\left\|\left\langle\left|\nabla_{x} v\right|\right\rangle\right\|_{L^{8}(\omega)} & \leqslant C\left\|\left\langle\left|\nabla_{x} v\right|\right\rangle\right\|_{L^{2}(\omega)}^{1 / 4}\left\|\left\langle\left|\nabla_{x} v\right|\right\rangle\right\|_{H^{1}(\omega)}^{3 / 4} \\
& \leqslant C\|v\|_{H^{1}(\Omega)}^{1 / 4}\left(\left\|n_{\mathrm{s}}\right\|_{L^{18 / 7}(\omega)}^{3 / 4}+\|v\|_{H^{1}(\Omega)}^{3 / 4}\right) .
\end{aligned}
$$

By interpolation inequalities, we get

$$
\left\|\frac{n_{\mathrm{s}}}{\sqrt{N_{\mathrm{S}}^{\infty}}}\right\|_{L^{3}(\omega)}^{2} \leqslant\left\|\frac{n_{\mathrm{s}}}{\sqrt{N_{\mathrm{S}}^{\infty}}}\right\|_{L^{2}(\omega)}\left\|\frac{n_{\mathrm{S}}}{\sqrt{N_{\mathrm{S}}^{\infty}}}\right\|_{L^{6}(\omega)}
$$

and

$$
\left\|n_{\mathrm{s}}\right\|_{L^{18 / 7}(\omega)} \leqslant\left\|n_{\mathrm{s}}\right\|_{L^{2}(\omega)}^{2 / 3}\left\|n_{\mathrm{s}}\right\|_{L^{6}(\omega)}^{1 / 3} .
$$

Thus, by (3.27) and (3.29)-(3.31), we obtain

$$
\begin{aligned}
\int_{\omega} \frac{\left(n_{\mathrm{s}}\right)^{2}}{N_{\mathrm{s}}^{\infty}} & \exp \left\{C_{2}\left(\left\|V^{\infty}\right\|_{L_{z}^{2}(0,1)}+\|v\|_{L_{z}^{2}(0,1)}\right)\right\}\left\langle\left|\nabla_{x} v\right|\right\rangle^{2} \mathrm{~d} x \\
& \leqslant C\left\|\frac{n_{\mathrm{s}}}{\sqrt{N_{\mathrm{s}}^{\infty}}}\right\|_{L^{2}(\omega)}\left\|\frac{n_{\mathrm{s}}}{\sqrt{N_{\mathrm{s}}^{\infty}}}\right\|_{L^{6}(\omega)}\|v\|_{H^{1}(\Omega)}^{1 / 2}\left(\left\|n_{\mathrm{s}}\right\|_{L^{2}(\omega)}\left\|n_{\mathrm{s}}\right\|_{L^{6}(\omega)}^{1 / 2}+\|v\|_{H^{1}(\Omega)}^{3 / 2}\right) .
\end{aligned}
$$

Finally, using $N_{\mathrm{s}}^{\infty} \geqslant C>0$ and Lemma 3.2, we have

$$
\begin{aligned}
& \int_{\omega} \frac{\left(n_{\mathrm{s}}\right)^{2}}{N_{\mathrm{S}}^{\infty}} \mathrm{e}^{C_{2}\left(\left\|V^{\infty}\right\|_{L_{z}^{2}(0,1)}+\|v\|_{L_{z}^{2}(0,1)}\right)}\left\langle\left|\nabla_{x} v\right|\right\rangle^{2} \mathrm{~d} x \\
& \quad \leqslant C_{1} L(t)\left\|n_{\mathrm{s}}\right\|_{L^{6}(\omega)}^{3 / 2}\|v\|_{H^{1}(\Omega)}^{1 / 2}+C_{2} L(t)^{1 / 2}\left\|n_{\mathrm{s}}\right\|_{L^{6}(\omega)}\|v\|_{H^{1}(\Omega)}^{2} .
\end{aligned}
$$

Now, to handle the term $\left\|n_{\mathrm{s}}\right\|_{L^{6}(\omega)}$, we decompose $\left\|n_{\mathrm{s}}\right\|_{L^{6}(\omega)} \leqslant C\left(\left\|n_{\mathrm{s}} / N_{\mathrm{s}}^{\infty}+v_{\mathrm{s}}\right\|_{L^{6}(\omega)}+\right.$ $\left.\left\|v_{\mathrm{s}}\right\|_{L^{6}(\omega)}\right)$. By (3.18) we have

$$
\left|v_{\mathrm{s}}\right| \leqslant \max \left\{\sum_{k} \frac{\mathrm{e}^{-\epsilon_{k}}}{\sum_{\ell} \mathrm{e}^{-\epsilon_{\ell}}}\left|\epsilon_{k}-\epsilon_{k}^{\infty}\right|, \sum_{k} \frac{\mathrm{e}^{-\epsilon_{k}^{\infty}}}{\sum_{\ell} \mathrm{e}^{-\epsilon_{\ell}^{\infty}}}\left|\epsilon_{k}^{\infty}-\epsilon_{k}\right|\right\} .
$$

Hence, with Lemma A 4 and (3.26), we deduce that

$$
\left\|v_{\mathbf{s}}\right\|_{L^{6}(\omega)} \leqslant C\|v\|_{L_{x}^{8} L_{z}^{1}(\Omega)} \leqslant C\|v\|_{H^{1}(\Omega)}
$$


due to the Sobolev embedding of $H^{1}(\Omega)$ into $L_{x}^{8} L_{z}^{1}(\Omega)$, which proves the inequality (3.13) in Lemma 3.4. Moreover, Proposition 3.1 provides a uniform bound on $\|v\|_{H^{1}(\Omega)}$ which, with the inequality (3.32) and Lemma 3.2, leads to

$$
\begin{aligned}
& \int_{\omega} \frac{\left(n_{\mathrm{s}}\right)^{2}}{N_{\mathrm{s}}^{\infty}} \exp \left\{C_{2}\left(\left\|V^{\infty}\right\|_{L_{z}^{2}(0,1)}+\|v\|_{L_{z}^{2}(0,1)}\right)\right\}\left\langle\left|\nabla_{x} v\right|\right\rangle^{2} \mathrm{~d} x \\
& \quad \leqslant C_{1} L(t)\left\|\frac{n_{\mathrm{s}}}{N_{\mathrm{S}}^{\infty}}+v_{\mathrm{s}}\right\|_{L^{6}(\omega)}^{3 / 2}+C_{2} L(t)^{1 / 2}\left\|\frac{n_{\mathrm{s}}}{N_{\mathrm{s}}^{\infty}}+v_{\mathrm{s}}\right\|_{L^{6}(\omega)}\|v\|_{H^{1}(\Omega)}+C_{3} L(t)\|v\|_{H^{1}(\Omega)} .
\end{aligned}
$$

Finally, using

$$
x^{1 / 4} y^{3 / 4} \leqslant \frac{1}{4 \varepsilon^{3}} x+\frac{3}{4} \varepsilon y,
$$

we have

$$
\begin{aligned}
L(t)\left\|\frac{n_{\mathrm{s}}}{N_{\mathrm{s}}^{\infty}}+v_{\mathrm{s}}\right\|_{L^{6}(\omega)}^{3 / 2} & \leqslant \frac{1}{4 \varepsilon^{3}} L(t)^{4}+\frac{3}{4} \varepsilon\left\|\frac{n_{\mathrm{s}}}{N_{\mathrm{s}}^{\infty}}+v_{\mathrm{s}}\right\|_{L^{6}(\omega)}^{2} \\
& \leqslant \frac{1}{4 \varepsilon^{3}} L(t)^{4}+C \varepsilon\left\|\nabla_{x}\left(\frac{n_{\mathrm{s}}}{N_{\mathrm{s}}^{\infty}}+v_{\mathrm{s}}\right)\right\|_{L^{2}(\omega)}^{2},
\end{aligned}
$$

where the Sobolev embedding $H^{1} \hookrightarrow L^{6}(\omega)$ and the Poincaré inequality are used. Proceeding analogously for the second term in (3.33), we obtain the desired inequality for $\varepsilon$ fixed small enough.

In order to estimate the second term in (3.25), we first use the Sobolev embedding $H^{1}(\Omega) \hookrightarrow L_{x}^{8} L_{z}^{1}(\Omega)$ and $(3.26)$, we have

$$
\begin{aligned}
& \int_{\omega} \frac{\left(n_{\mathrm{s}}\right)^{2}}{N_{\mathrm{s}}^{\infty}} \exp \left\{C_{2}\left(\left\|V^{\infty}\right\|_{L_{z}^{2}(0,1)}+\|v\|_{L_{z}^{2}(0,1)}\right)\right\}\langle|v|\rangle^{2}\left\langle\left|\nabla_{x} V^{\infty}\right|\right\rangle^{2} \mathrm{~d} x \\
& \leqslant C\|v\|_{H^{1}(\Omega)}^{2}\left\|\frac{n_{\mathrm{s}}}{\sqrt{N_{\mathrm{s}}^{\infty}}}\right\|_{L^{3}(\omega)}^{2} .
\end{aligned}
$$

With (3.30) and Lemma 3.2, it yields

$$
\begin{aligned}
\int_{\omega} \frac{\left(n_{\mathrm{s}}\right)^{2}}{N_{\mathrm{S}}^{\infty}} \exp \left\{C_{2}\left(\left\|V^{\infty}\right\|_{L_{z}^{2}(0,1)}+\|v\|_{L_{z}^{2}(0,1)}\right)\right\}\langle|v|\rangle^{2}\left\langle\left|\nabla_{x} V^{\infty}\right|\right\rangle^{2} \mathrm{~d} x \\
\leqslant C L(t)^{1 / 2}\|v\|_{H^{1}(\Omega)}^{2}\left(\left\|\frac{n_{\mathrm{s}}}{N_{\mathrm{s}}^{\infty}}+v_{\mathrm{s}}\right\|_{L^{6}(\omega)}+\left\|v_{\mathrm{s}}\right\|_{L^{6}(\omega)}\right) .
\end{aligned}
$$

By proceeding as above, we obtain the desired inequality for the second term, which concludes the proof.

Acknowledgements. The authors acknowledge support by the European IHP Network grant (no. RNT2 2001 349) 'Hyperbolic and kinetic equation: asymptotics, numerics, applications', by the ACI Nouvelles Interfaces des Mathématiques Grant (no. ACINIM 176-2004) 'MOQUA', funded by the French Ministry of Research, and by ACI Jeunes Chercheurs grant (no. JC1035) 'Modèles dispersifs vectoriels pour le transport à l'échelle nanométrique'. 


\section{Appendix A. Spectral properties of the Hamiltonian}

In this appendix, we first list some basic properties of eigenfunctions and eigenvalues of the Schrödinger operator in the $z$ variable. Most of these properties, which are used throughout the paper, are either proven or can be proved by straightforwardly adapting the techniques in $[\mathbf{3 0}]$. Therefore, very few proofs are provided in this appendix.

For a given real-valued function $U$ in $L^{2}(0,1)$, let $H[U]$ be the Schrödinger operator

$$
H[U]:=-\frac{1}{2} \frac{\mathrm{d}^{2}}{\mathrm{~d} z^{2}}+U(z)
$$

defined on the domain $D(H[U])=H^{2}(0,1) \cap H_{0}^{1}(0,1)$.

This operator admits a strictly increasing sequence of real eigenvalues $\left(\epsilon_{k}[U]\right)_{k \geqslant 1}$ going to $+\infty$. The corresponding eigenvectors, denoted by $\left(\chi_{k}[U](z)\right)_{k \geqslant 1}$ (chosen such that $\left.\chi_{k}^{\prime}(0)>0\right)$, form an orthonormal basis of $L^{2}(0,1)$. They, of course, satisfy

$$
\left.\begin{array}{c}
-\frac{1}{2} \frac{\mathrm{d}^{2}}{\mathrm{~d} z^{2}} \chi_{k}+U \chi_{k}=\epsilon_{k} \chi_{k}, \\
\chi_{k} \in H_{0}^{1}(0,1), \quad \int_{0}^{1} \chi_{k} \chi_{\ell} \mathrm{d} z=\delta_{k l} .
\end{array}\right\}
$$

Obviously, for $U=0$, we have $\epsilon_{k}[0]=\frac{1}{2} \pi^{2} k^{2}$ and $\chi_{k}[0](z)=\sqrt{2} \sin (\pi k z)$.

Lemma A 1. Let $U$ and $V$ be two real-valued functions in $L^{2}(0,1)$ such that $U-V \in$ $L^{\infty}(0,1)$. Then the corresponding eigenvalues verify

$$
\left|\epsilon_{k}[U]-\epsilon_{k}[V]\right| \leqslant\|U-V\|_{L^{\infty}(0,1)} .
$$

In particular, the case $V=0$ gives $\left|\epsilon_{k}[U]-\frac{1}{2} \pi^{2} k^{2}\right| \leqslant\|U\|_{L^{\infty}(0,1)}$.

Moreover, following the study of the spectral properties of $H[U]$ in [30, Chapter 2], we have the following lemma.

Lemma A 2. There exists a positive constant $C_{U}$ depending only on $\|U\|_{L^{2}(0,1)}$ such that

$$
\left|\epsilon_{k}[U]-\frac{1}{2} \pi^{2} k^{2}\right| \leqslant C_{U} \quad \text { and } \quad\left\|\chi_{k}[U]-\sqrt{2} \sin (\pi k z)\right\|_{L^{\infty}(0,1)} \leqslant C_{U} .
$$

Moreover, the constant $C_{U}$ can be chosen such that $C_{U} \leqslant C_{1} \exp \left(C_{2}\|U\|_{L^{2}(0,1)}\right)$, where the constants $C_{1}$ and $C_{2}$ are independent of $U$ and $k$.

Lemma A 3. Let $V=V(\lambda, z) \in L_{\mathrm{loc}}^{\infty}\left(\lambda, L_{z}^{2}(0,1)\right)$, where $\lambda$ is a real parameter (typically $\lambda=t$ or $\left.\lambda=x_{i}\right)$. Let us define $\epsilon_{k}$ as shorthand for $\epsilon_{k}[V(\lambda, \cdot)]$ and $\chi_{k}$ for $\chi_{k}[V(\lambda, \cdot)]$. Assume that $\partial_{\lambda} V \in L_{\text {loc }}^{1}\left(\lambda, L_{z}^{2}(0,1)\right)$. Then

(i) $\partial_{\lambda} \epsilon_{k} \in L_{\text {loc }}^{1}$ and

$$
\partial_{\lambda} \epsilon_{k}=\left\langle\left|\chi_{k}\right|^{2} \partial_{\lambda} V\right\rangle
$$

(ii) $\partial_{\lambda} \chi_{k} \in L_{\text {loc }}^{1}\left(\lambda, L_{z}^{\infty}(0,1)\right)$ and we have

$$
\partial_{\lambda} \chi_{k}=\sum_{\ell \neq k} \frac{\left\langle\chi_{k} \chi_{\ell} \partial_{\lambda} V\right\rangle}{\epsilon_{k}-\epsilon_{\ell}} \chi_{\ell}
$$


Lemma A 4. Let $V$ and $\tilde{V}$ be two real-valued functions in $L^{2}(0,1)$. There then exist two positive constants $C_{1}$ and $C_{2}$ independent of $p, V$ and $\tilde{V}$ such that

$$
\left|\epsilon_{k}[V]-\epsilon_{k}[\tilde{V}]\right| \leqslant C_{1} \exp \left(C_{2}\left(\|V\|_{L^{2}(0,1)}+\|\tilde{V}\|_{L^{2}(0,1)}\right)\right)\|V-\tilde{V}\|_{L^{1}(0,1)}
$$

and

$$
\left\|\chi_{k}[V]-\chi_{k}[\tilde{V}]\right\|_{L^{\infty}(0,1)} \leqslant C_{1} \exp \left(C_{2}\left(\|V\|_{L^{2}(0,1)}+\|\tilde{V}\|_{L^{2}(0,1)}\right)\right)\|V-\tilde{V}\|_{L^{1}(0,1)} .
$$

Proof. The estimate (A 3) is an easy consequence of Lemmas A 2 and A 3.

Let us prove (A 4). Without loss of generality, we assume that $\epsilon_{k}[V]>0$ (by shifting $V$ and $\tilde{V}$ by the same constant). Let us define

$$
u_{k}=\frac{\chi_{k}[\tilde{V}]^{\prime}(0)}{\chi_{k}[V]^{\prime}(0)} \chi_{k}[V] \quad \text { and } \quad \tilde{u}_{k}=\chi_{k}[\tilde{V}]
$$

so that $u_{k}^{\prime}(0)=\tilde{u}_{k}^{\prime}(0)$. Writing the equation satisfied by $u_{k}-\tilde{u}_{k}$ and proceeding as in the proof of [30, Lemma 1, Chapter 1], we have

$$
\begin{aligned}
u_{k}(z)-\tilde{u}_{k}(z)=2 \int_{0}^{z} s(z-t) & V(t)\left(u_{k}-\tilde{u}_{k}\right)(t) \mathrm{d} t \\
& +2 \int_{0}^{z} s(z-t) \tilde{u}_{k}(t)\left((V-\tilde{V})(t)-\left(\epsilon_{k}[V]-\epsilon_{k}[\tilde{V}]\right)\right) \mathrm{d} t
\end{aligned}
$$

where

$$
s(t)=\frac{\sin \left(\sqrt{2 \epsilon_{k}[V]} t\right)}{\sqrt{2 \epsilon_{k}[V]}} .
$$

By a Gronwall argument, we prove (A 4) for the difference $u_{k}-\tilde{u}_{k}$. We finally deduce the result for $\chi_{k}[V]-\chi_{k}[\tilde{V}]$ by using the property

$$
\int_{0}^{1}\left|\chi_{k}\right|^{2} \mathrm{~d} z=1
$$

We now give two technical lemmas, where the potential is defined on $\Omega$. We recall that $(x, z) \in \Omega=\omega \times(0,1)$, where $\omega$ is a bounded regular domain of $\mathbb{R}^{2}$.

Lemma A 5. Assume that $V \in H^{1}(\Omega)$ and let $\epsilon_{k}$ be the eigenvalues defined by (1.2). Then, for all $\alpha \geqslant 0$ and $q \in[1,+\infty)$, we have

$$
I_{\alpha}:=\frac{1}{\mathcal{Z}} \sum_{k}\left|\epsilon_{k}\right|^{\alpha} \mathrm{e}^{-\epsilon_{k}} \in L^{q}(\omega),
$$

where $\mathcal{Z}=\sum_{k} \mathrm{e}^{-\epsilon_{k}}$. The $L^{q}$ norm of $I_{\alpha}$ is bounded by a constant depending only on $\alpha$, $q$ and $\|V\|_{H^{1}}$. 
Proof. Lemma A 2 states that the eigenvalues and eigenvectors of (1.2) satisfy the (uniform-in- $p$ ) estimate

$$
\left|\epsilon_{k}(x)-\frac{1}{2} \pi^{2} k^{2}\right|+\left\|\chi_{k}(x, \cdot)\right\|_{L_{z}^{\infty}} \leqslant C_{1} \exp \left\{C_{2}\|V(x, \cdot)\|_{L_{z}^{2}}\right\} .
$$

It is sufficient to show that

$$
I_{\alpha}(x) \leqslant C_{3} \exp \left\{\alpha C_{2}\|V(x, \cdot)\|_{L_{z}^{2}}\right\} .
$$

Indeed, since $\|V(x, \cdot)\|_{L_{z}^{2}}$ is bounded in $H^{1}(\omega)$, the Trudinger inequality

$$
\int_{\mathcal{O}} \exp \left(|u|^{N /(N-1)}\right)<+\infty, \quad \forall u \in W^{1, N}(\mathcal{O}), \mathcal{O} \subset \mathbb{R}^{N}
$$

implies that $\exp \left\{\|V(x, \cdot)\|_{L_{2}^{2}}^{2}\right\} \in L^{1}(\omega)$, which ensures that $\exp \left\{\alpha C_{2}\|V(x, \cdot)\|_{L_{z}^{2}}\right\} \in L^{q}(\omega)$ for all $q<+\infty$, which thus leads to the result.

Let us now prove (A 7). To this aim, we treat low and high energies differently. More precisely, we have

$$
\begin{aligned}
I_{\alpha} & =\frac{1}{\mathcal{Z}} \sum_{\left|\epsilon_{k}\right| \leqslant K A}\left|\epsilon_{k}\right|^{\alpha} \mathrm{e}^{-\epsilon_{k}}+\frac{1}{\mathcal{Z}} \sum_{\left|\epsilon_{k}\right| \geqslant K A}\left|\epsilon_{k}\right|^{\alpha} \mathrm{e}^{-\epsilon_{k}} \\
& \leqslant(K A)^{\alpha}+\frac{1}{\mathcal{Z}} \sum_{\left|\epsilon_{k}\right| \geqslant K A}\left|\epsilon_{k}\right|^{\alpha} \mathrm{e}^{-\epsilon_{k}}
\end{aligned}
$$

where we choose $K>2$, and $A$ is such that $\left|\epsilon_{k}-\frac{1}{2} \pi^{2} k^{2}\right|<A$. This choice implies that

$$
\frac{1}{2} k^{2} \pi^{2}-A<\epsilon_{k}<\frac{1}{2} k^{2} \pi^{2}+A
$$

and, for high energies $\left(\left|\epsilon_{k}\right| \geqslant K A\right)$, that we have

$$
A<\frac{1}{2(K-1)} k^{2} \pi^{2} .
$$

Hence, the high-energy contribution can be estimated as follows:

$$
\begin{aligned}
\sum_{\left|\epsilon_{k}\right| \geqslant K A}\left|\epsilon_{k}\right|^{\alpha} \mathrm{e}^{-\epsilon_{k}} & \leqslant \sum_{k>\sqrt{2(K-1) A} / \pi}\left(\frac{1}{2} k^{2} \pi^{2}+A\right)^{\alpha} \mathrm{e}^{-k^{2} \pi^{2} / 2} \mathrm{e}^{A} \\
& \leqslant\left(1+\frac{1}{K-1}\right)^{\alpha} \mathrm{e}^{A} \sum_{k>\sqrt{2(K-1) A} / \pi}\left(\frac{1}{2} k^{2} \pi^{2}\right)^{\alpha} \mathrm{e}^{-k^{2} \pi^{2} / 2} \\
& \leqslant C_{\alpha}\left(1+\frac{1}{K-1}\right)^{\alpha} \mathrm{e}^{A} \int_{\sqrt{2(K-1) A} / \pi}^{\infty}\left(\frac{1}{2} \pi^{2} x^{2}\right)^{\alpha} \mathrm{e}^{-\pi^{2} x^{2} / 2} \mathrm{~d} x
\end{aligned}
$$

where we used the elementary property

$$
\lim _{n \rightarrow+\infty} \frac{\sum_{k \geqslant n} f(k)}{\int_{n}^{+\infty} f(x) \mathrm{d} x}=1,
$$


for any non-negative function decaying at infinity and such that the following integral $\int_{0}^{+\infty} f(x) \mathrm{d} x$ converges. Assuming that $\alpha \neq 0$ (the case $\alpha=0$ is trivial), an integration by parts leads to the estimate

$$
\int_{\sqrt{2(K-1) A} / \pi}^{\infty}\left(\frac{1}{2} \pi^{2} x^{2}\right)^{\alpha} \mathrm{e}^{-\pi^{2} x^{2} / 2} \mathrm{e}^{A} \mathrm{~d} x \leqslant C((K-1) A)^{\alpha-(1 / 2)} \mathrm{e}^{-(K-1) A} \mathrm{e}^{A},
$$

which leads to

$$
\sum_{\left|\epsilon_{k}\right| \geqslant K A}\left|\epsilon_{k}\right|^{\alpha} \mathrm{e}^{-\epsilon_{k}} \leqslant C_{\alpha} A^{\alpha-(1 / 2)} \mathrm{e}^{-(K-1) A} \mathrm{e}^{A} .
$$

Besides, due to the choice of $A$, we obviously have

$$
\sum_{k} \mathrm{e}^{-\epsilon_{k}} \geqslant C \mathrm{e}^{-A}
$$

Therefore, returning to (A 9), we have

$$
I_{\alpha} \leqslant(K A)^{\alpha}+C_{\alpha} A^{\alpha-(1 / 2)} \mathrm{e}^{(3-K) A} .
$$

Setting $K=4$, we obtain a bound on $A^{-1 / 2} \mathrm{e}^{(3-K) A}$ for large $A$. Thus, we have proved that $I \leqslant C_{\alpha} A^{\alpha}$ and (A 7) follows due to (A 6) by taking $A=C_{1} \exp \left(C_{2}\|V(x, \cdot)\|_{L_{z}^{2}}\right)$.

Lemma A 6. The map

$$
V \mapsto V_{\mathrm{s}}=-\log \left(\sum_{k} \mathrm{e}^{-\epsilon_{k}[V]}\right)
$$

is locally Lipschitz continuous from $H^{2}(\Omega)$ to $H^{2}(\omega)$, where $\left(\epsilon_{k}[V]\right)_{k}$ denotes the whole set of eigenvalues of the Hamiltonian

$$
-\frac{1}{2} \frac{\mathrm{d}^{2}}{\mathrm{~d} z^{2}}+V
$$

Proof. Since the summation over $k$ can be done easily, it is enough to show the result for the map $V \mapsto \epsilon_{k}[V]$. Let $U$ and $V$ be two bounded potentials of $H^{2}(\Omega)$. From Lemma A 1, we easily deduce that $\left\|\epsilon_{k}[U]-\epsilon_{k}[V]\right\|_{L^{2}(\omega)} \leqslant C\|U-V\|_{H^{2}(\Omega)}$. For the first derivative, with Lemma A 3, we write

$$
\begin{aligned}
\int_{\omega}\left|\nabla_{x} \epsilon_{k}[U]-\nabla_{x} \epsilon_{k}[V]\right|^{2} \mathrm{~d} x \leqslant 2 \int_{\Omega} \mid \nabla_{x}( & U-V)\left.\right|^{2}\left|\chi_{k}[U]\right|^{4} \mathrm{~d} x \mathrm{~d} z \\
& +2 \int_{\Omega}\left|\nabla_{x} V\right|^{2}\left(\left|\chi_{k}[U]\right|^{2}-\left|\chi_{k}[V]\right|^{2}\right)^{2} \mathrm{~d} x \mathrm{~d} z .
\end{aligned}
$$

The Sobolev embedding of $H^{2}(\Omega)$ into $L^{\infty}(\Omega)$ implies that, for all non-negative constants $C_{2}$,

$$
\exp \left(C_{2}\left(\|U\|_{L_{z}^{2}(0,1)}+\|V\|_{L_{z}^{2}(0,1)}\right)\right) \in L^{\infty}(\omega)
$$


Thus, with Lemma A 2 we have a bound of $\chi_{k}[U]$ in $L^{\infty}(\Omega)$ and with Lemma A 4 we have

$$
\left\|\chi_{k}[U]-\chi_{k}[V]\right\|_{L^{\infty}(\Omega)} \leqslant C\|U-V\|_{H^{2}(\Omega)} .
$$

We deduce then that

$$
\int_{\omega}\left|\nabla_{x} \epsilon_{k}[U]-\nabla_{x} \epsilon_{k}[V]\right|^{2} \mathrm{~d} x \leqslant C\|U-V\|_{H^{1}(\Omega)}^{2}+C\|U-V\|_{H^{2}(\Omega)}^{2} .
$$

It now remains to estimate the second derivative of $\epsilon_{k}[U]-\epsilon_{k}[V]$. We recall that, from Lemma A 1, we may easily deduce that

$$
\left\|\epsilon_{k}[U]-\epsilon_{k}[V]\right\|_{L^{\infty}(\omega)} \leqslant C\|U-V\|_{H^{2}(\Omega)} .
$$

If $i=1$ or $2, j=1$ or 2 , by the expression of the derivatives stated in Lemma A 3 we have

$$
\partial_{x_{i} x_{j}} \epsilon_{k}[V]=\int_{0}^{1} \partial_{x_{i} x_{j}} V\left|\chi_{k}[V]\right|^{2} \mathrm{~d} z+2 \int_{0}^{1} \chi_{k}[V] \partial_{x_{i}} \chi_{k}[V] \partial_{x_{j}} V \mathrm{~d} z .
$$

As before, we can show the Lipschitz dependency in $V \in H^{2}(\Omega)$ of the first term on the right-hand side. For the second term, we need the following result, which is proved below: there exists a positive constant $\delta_{V}$ depending only on $\|V\|_{H^{2}(\Omega)}$ such that

$$
\forall(k, l) \in\left(\mathbb{N}^{*}\right)^{2}, \quad\left|\epsilon_{k}[V]-\epsilon_{\ell}[V]\right| \geqslant \delta_{V}|k-l|^{2} .
$$

Since $\chi_{k}[V]$ is bounded in $L^{\infty}(\Omega)$, using the expression of $\partial_{x_{i}} \chi_{k}[V]$ in Lemma A 3 and (A 12), we obtain $\left|\partial_{x_{i}} \chi_{k}[V]\right| \leqslant C\left\langle\left|\partial_{x_{i}} V\right|\right\rangle$. Therefore,

$$
\begin{aligned}
\mid \chi_{k}[U] \partial_{x_{i}} \chi_{k}[U] \partial_{x_{j}} U & -\chi_{k}[V] \partial_{x_{i}} \chi_{k}[V] \partial_{x_{j}} V \mid \\
\leqslant & C\left|\chi_{k}[U]-\chi_{k}[V]\right|\left\langle\left|\partial_{x_{i}} U\right|\right\rangle\left|\partial_{x_{i}} U\right| \\
& +C\left|\partial_{x_{i}} U\right|\left|\partial_{x_{i}} \chi_{k}[U]-\partial_{x_{i}} \chi_{k}[V]\right|+C\left\langle\left|\partial_{x_{i}} V\right|\right\rangle\left|\partial_{x_{i}}(U-V)\right| .
\end{aligned}
$$

Thus, it remains to see the Lipschitz dependency in $V$ of $\partial_{x_{i}} \chi_{k}[V]$. We have

$$
\begin{aligned}
\partial_{x_{i}}\left(\chi_{k}[U]-\chi_{k}[V]\right)= & \sum_{\ell \neq k}\left(\frac{\left\langle\chi_{k}[U] \chi_{\ell}[U] \partial_{x_{i}} U\right\rangle}{\epsilon_{k}[U]-\epsilon_{\ell}[U]} \chi_{\ell}[U]-\frac{\left\langle\chi_{k}[V] \chi_{\ell}[V] \partial_{x_{i}} V\right\rangle}{\epsilon_{k}[V]-\epsilon_{\ell}[V]} \chi_{\ell}[V]\right) \\
= & \sum_{\ell \neq k} \frac{\left\langle\chi_{k}[U] \chi_{\ell}[U] \partial_{x_{i}} U-\chi_{k}[V] \chi_{\ell}[V] \partial_{x_{i}} V\right\rangle}{\epsilon_{k}[U]-\epsilon_{\ell}[U]} \chi_{\ell}[U] \\
& +\sum_{\ell \neq k} \frac{\left\langle\chi_{k}[V] \chi_{\ell}[V] \partial_{x_{i}} V\right\rangle}{\epsilon_{k}[V]-\epsilon_{\ell}[V]}\left(\chi_{\ell}[U]-\chi_{\ell}[V]\right) \\
& +\sum_{\ell \neq k}\left\langle\chi_{k}[V] \chi_{\ell}[V] \partial_{x_{i}} V\right\rangle \chi_{\ell}[V] \frac{\epsilon_{k}[V]-\epsilon_{k}[U]+\epsilon_{\ell}[U]-\epsilon_{\ell}[V]}{\left(\epsilon_{k}[U]-\epsilon_{\ell}[U]\right)\left(\epsilon_{k}[V]-\epsilon_{\ell}[V]\right)} .
\end{aligned}
$$

From (A 10)-(A 12), we deduce that

$$
\left\|\partial_{x_{i}} \chi_{k}[U]-\partial_{x_{i}} \chi_{k}[V]\right\|_{L^{2}(\Omega)} \leqslant C\left(1+\left\|\partial_{x_{i}} U\right\|_{L^{2}(\Omega)}+\left\|\partial_{x_{i}} V\right\|_{L^{2}(\Omega)}\right)\|U-V\|_{H^{2}(\Omega)} .
$$

With the Sobolev embedding of $H^{1}(\Omega)$ into $L^{2}(\Omega),\left\|\partial_{x_{i}} V\right\|_{L^{2}(\Omega)} \leqslant C\|V\|_{H^{2}(\Omega)}$. This concludes the proof of the Lipschitz dependency of the second derivative with respect to $V$. 
Proof of (A 12). If $k=\ell$, this inequality is obvious. Let us first prove that there exists a constant, $\delta_{V}$, depending only on $\|V\|_{H^{2}(\Omega)}$, such that

$$
\min _{k \neq l}\left|\epsilon_{k}[V]-\epsilon_{\ell}[V]\right| \geqslant \delta_{V}
$$

If not, by the compact embedding of $H^{2}(\Omega)$ into $L^{\infty}(\Omega)$ it would be possible to find a sequence $\left(V^{n}\right)$ converging to $V$ in the $L^{\infty}$ strong topology and a sequence $k^{n}$ of integers such that $\epsilon_{k^{n}+1}\left[V^{n}\right]-\epsilon_{k^{n}}\left[V^{n}\right]$ converges to zero as $n \rightarrow+\infty$. The asymptotic behaviour of the $\epsilon_{k}$ deduced from Lemma A 1 implies that the sequence $\left(k^{n}\right)$ is bounded; thus, up to an extraction, it is stationary: $k^{n}=k$. Besides, (A 2) implies that $\epsilon_{k}\left[V^{n}\right]$ converges to $\epsilon_{k}[V]$ and $\epsilon_{k+1}\left[V^{n}\right]$ to $\epsilon_{k+1}[V]$. Hence, $\epsilon_{k}[V]=\epsilon_{k+1}[V]$, which is a contradiction of the fact that the eigenvalues are strictly increasing. Moreover, by (A 2), we have

$$
\frac{1}{2} \pi^{2} k^{2}-\|V\|_{L^{\infty}(\Omega)} \leqslant \epsilon_{k}[V] \leqslant \frac{1}{2} \pi^{2} k^{2}+\|V\|_{L^{\infty}(\Omega)} .
$$

Therefore, for any $(k, l)$,

$$
\left|\epsilon_{k}[V]-\epsilon_{\ell}[V]\right| \geqslant \frac{1}{2} \pi^{2}|k-\ell|^{2}+\pi^{2}|k-\ell|-2\|V\|_{L^{\infty}(\Omega)} .
$$

Hence, if $\pi^{2}|k-\ell| \geqslant 2\|V\|_{L^{\infty}(\Omega)}$, , then $\left|\epsilon_{k}[V]-\epsilon_{\ell}[V]\right| \geqslant \frac{1}{2} \pi^{2}|k-\ell|^{2}$. From this inequality and (A 13) we easily deduce (A 12) (up to a change of $\delta_{V}$ ).

\section{References}

1. T. Ando, B Fowler And F. Stern, Electronic properties of two-dimensional systems, Rev. Mod. Phys. 54 (1982), 437-672.

2. A. Arnold, P. Markowich and G. Toscani, On large time asymptotics for driftdiffusion-Poisson systems, Transport Theory Statist. Phys. 29 (2000), 571-581.

3. A. Arnold, P. Markowich, G. Toscani and A. Unterreiter, On generalized Csiszár-Kullback inequalities, Monatsh. Math. 131(3) (2000), 235-253.

4. F. Balestra, S. Cristoloveanu, M. Benachir, J. Brini and T. Elewa, Double-gate silicon-on-insulator transistor with volume inversion: a new device with greatly enhanced performance, IEEE Electron. Device Lett. 8 (1987), 410-412.

5. G. BASTARD, Wave mechanics applied to semiconductor heterostructures (Les Éditions de Physique, Les Ulis, 1996).

6. N. Ben Abdallah and F. MÉhats, On a Vlasov-Schrödinger-Poisson model, Commun. PDEs 29 (2004), 173-206.

7. N. Ben Abdallah And F. MÉhats, Semiclassical analysis of the Schrödinger equation with a partially confining potential, J. Math. Pures Appl. 84 (2005), 580-614.

8. N. Ben Abdallah, F. Méhats and G. Quinio, Global existence of classical solutions for a Vlasov-Schrödinger-Poisson system, Indiana Univ. Math. J., in press.

9. P. Biler And J. Dolbeault, Long time behavior of solutions of Nernst-Planck and Debye-Hückel drift-diffusion systems, Annls Inst. H. Poincaré 1 (2000), 461-472.

10. P. Biler, J. Dolbeault and P. A. Markowich, Large time asymptotics of nonlinear drift-diffusion systems with Poisson coupling, Transport Theory Statist. Phys. 30 (2001), 521-536.

11. I. CSISZÁR, Information-type measures of difference of probability distributions, Studia Sci. Math. Hungar. 2 (1967), 299-318. 
12. J. H. DAVIES, The physics of low dimensional semiconductors (Cambridge University Press, 1998).

13. P. Degond And C. Ringhofer, Quantum moment hydrodynamics and the entropy principle, J. Statist. Phys. 112 (2003), 587-628.

14. P. Degond, F. MÉHAts And C. Ringhofer, Quantum energy-transport and driftdiffusion models, J. Statist. Phys. 118 (2005), 625-665.

15. P. Degond, F. MÉhats And C. Ringhofer, Quantum hydrodynamic models derived from entropy principle, in Nonlinear partial differential equations and related analysis, pp. 107-131, Contemporary Mathematics, Volume 371 (American Mathematical Society, Providence, RI, 2005).

16. J. Dolbeault And M. Del Pino, Generalized Sobolev inequalities and asymptotic behaviour in fast diffusion and porous medium problems, preprint no. 9905, Centre De Recherche en Mathématiques de la Décision, Paris (1999).

17. D. K. FERRY AND S. M. GoODNICK, Transport in nanostructures (Cambridge University Press, 1997).

18. H. GAJEWSKI, On existence, uniqueness and asymptotic behavior of solutions of the basic equations for carrier transport in semiconductors, Z. Angew. Math. Mech. 65 (1985), 101108.

19. H. GAJEWSKI AND K. GRÖGER, Semiconductor equations for variable mobilities based on Boltzmann statistics or Fermi-Dirac statistics, Math. Nachr. 140 (1989), 7-36.

20. S. Gallego And F. MÉHats, Entropic discretization of a quantum drift-diffusion model, SIAM J. Numer. Analysis 43 (2005), 1828-1849.

21. F. Golse And F. Poupaud, Limite fluide des équation de Boltzmann des semiconducteurs pour une statistique de Fermi-Dirac, Asymp. Analysis 6 (1992), 135-169.

22. G. A. Hagedorn And A. Joye, A time-dependent Born-Oppenheimer approximation with exponentially small error estimates, Commun. Math. Phys. 223 (2001), 583-626.

23. S. KULLBACK, Information theory and statistics (Wiley, 1959).

24. O. A. Ladyzenskaja, V. A. Solonnikov and N. N. Ural'Ceva, Linear and quasilinear equations of parabolic type, Translations of Mathematical Monographs, Volume 23 (American Mathematical Society, Providence, RI, 1988).

25. P. A. Markowich, C. A. Ringhofer And C. Schmeiser, Semiconductor equations (Springer, 1990).

26. M. S. Mock, Analysis of mathematical models of semiconductor devices, Advances in Numerical Computation Series, Volume 3 (Boole Press, Dublin, 1983).

27. F. NIER, A stationary Schrödinger-Poisson system arising from the modelling of electronic devices, Forum Math. 2 (1990), 489-510.

28. F. NIER, Schrödinger-Poisson systems in dimension $d \leqslant 3$ : the whole-space case, Proc. R. Soc. Edinb. A 123 (1993), 1179-1201.

29. F. NIER, A variational formulation of Schrödinger-Poisson systems in dimension $d \leqslant 3$, Commun. PDEs 18 (1993), 1125-1147.

30. J. Pöschel And E. Trubowitz, Inverse spectral theory (Academic, 1987).

31. F. Poupaud, Diffusion approximation of the linear semiconductor Boltzmann equation: analysis of boundary layers, Asymp. Analysis 4 (1991), 293-317.

32. K. SEEGER, Semiconductor physics: an introduction, 6th edn (Springer, 1997).

33. H. Spohn and S. Teufel, Adiabatic decoupling and time-dependent Born-Oppenheimer theory, Commun. Math. Phys. 224 (2001), 113-132.

34. S. Teufel, Adiabatic perturbation theory in quantum dynamics, Lecture Notes in Mathematics, Volume 1821 (Springer, 2003).

35. B. Vinter And C. Weisbuch, Quantum semiconductor structures (Academic, 1991). 